\title{
Mechanism of Boron-Catalyzed N-Alkylation of Amines with Carboxylic Acids
}

\author{
Qi Zhang, ${ }^{a}$ Ming-Chen Fu, ${ }^{a}$ Hai-Zhu Yu, ${ }^{* b}$ Yao Fu ${ }^{* a}$ \\ ${ }^{a}$ Hefei National Laboratory for Physical Sciences at the Microscale, iChEM, CAS Key \\ Laboratory of Urban Pollutant Conversion, Anhui Province Key Laboratory of Biomass Clean \\ Energy, Department of Chemistry, University of Science and Technology of China, Hefei 230026, \\ People's Republic of China \\ ${ }^{b}$ Department of Chemistry and Centre for Atomic Engineering of Advanced Materials, Anhui \\ University, Hefei, 230601 \\ Emails: yuhaizhu@ahu.edu.cn; fuyao@ustc.edu.cn
}

\section{Table of Contents}

Details of the control experiments. .S2

Gibbs Free Energy Corrections and Electronic Energies in Solvate of related compounds and Transition States. .S3 
Details of the control experiments

\section{Table S1}

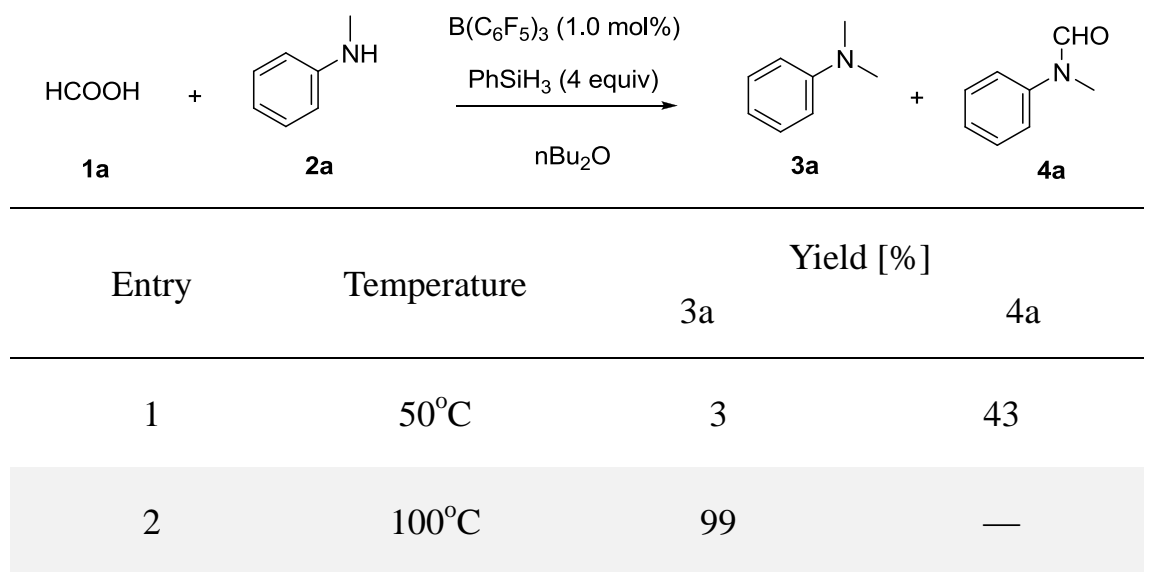

Note: Reaction conditions: $\mathrm{N}$-methylaniline $(0.2 \mathrm{mmol}), \mathrm{HCO}_{2} \mathrm{H}(2.3$ equiv), solvent $(1.0 \mathrm{~mL}), 8 \mathrm{~h}$ The yield is determined by GC analysis of the reaction mixture after quenching with aqueous $\mathrm{NaOH}$ solution. n-Dodecane was used as an internal standard.

\section{Table S2}

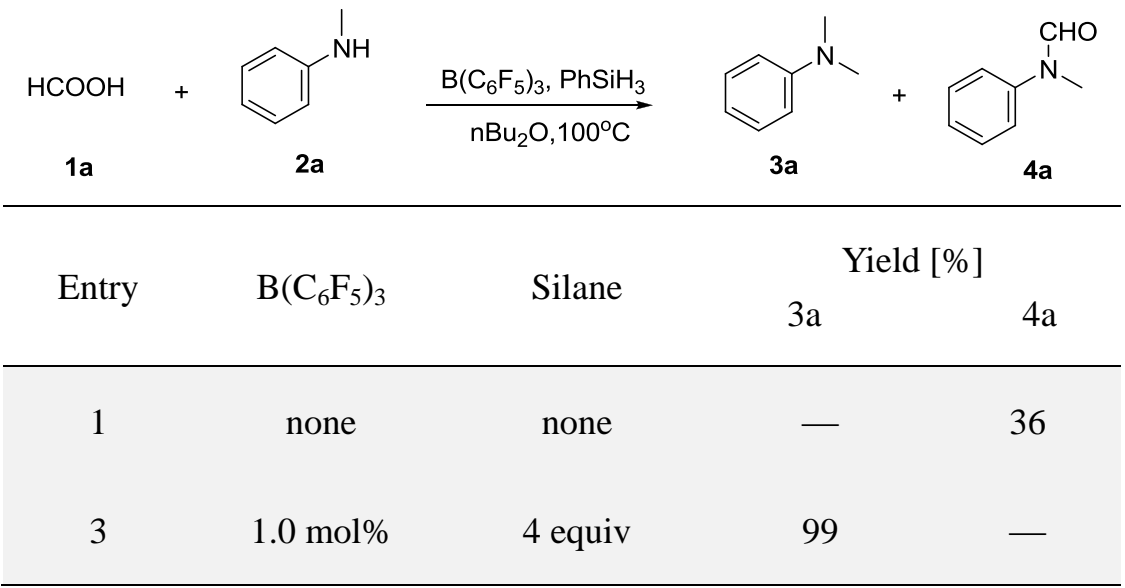

Note: Reaction conditions: $\mathrm{N}$-methylaniline $(0.2 \mathrm{mmol}), \mathrm{HCO}_{2} \mathrm{H}$ (2.3 equiv), solvent $(1.0 \mathrm{~mL})$, $100^{\circ} \mathrm{C}, 8 \mathrm{~h}$. The yield is determined by GC analysis of the reaction mixture after quenching with aqueous $\mathrm{NaOH}$ solution. n-Dodecane was used as an internal standard. 
Gibbs Free Energy Corrections and Electronic Energies in Solvate of related compounds and Transition States

\begin{tabular}{|c|c|c|}
\hline & $\begin{array}{c}\text { Thermal Correction of Gibbs } \\
\text { Free Energies (Hartree) }\end{array}$ & $\begin{array}{c}\text { Electronic Energy in solvent } \\
\text { (Hartree) }\end{array}$ \\
\hline $\mathrm{B}\left(\mathrm{C}_{6} \mathrm{~F}_{5}\right)_{3}$ & 0.091282 & -2208.215523 \\
\hline 1a & 0.009855 & -189.7543175 \\
\hline $2 a$ & 0.114666 & -326.8570319 \\
\hline $1 \mathrm{a}-\mathrm{B}$ & 0.125795 & -2397.995889 \\
\hline $2 \mathrm{a}-\mathrm{B}$ & 0.236769 & -2535.122908 \\
\hline $12 \mathrm{a}-\mathrm{B}$ & 0.261713 & -2724.89103793 \\
\hline SiOSi & 0.174371 & -1119.895439 \\
\hline $3 a$ & 0.140886 & -366.1504479 \\
\hline $\mathrm{H}_{2}$ & -0.001353 & -1.167905082 \\
\hline $\mathrm{HOSiH}_{2} \mathrm{Ph}$ & 0.088976 & -598.1564103 \\
\hline $4 a$ & 0.121735 & -440.1930918 \\
\hline $\mathrm{H}_{2} \mathrm{O}$ & 0.003381 & -76.42620134 \\
\hline Int1 & 0.040409 & -379.5295229 \\
\hline Int2 & 0.146112 & -516.609167 \\
\hline Int3 & 0.228489 & -2920.872731 \\
\hline Int4 & 0.0963 & -711.4841196 \\
\hline Int5 & 0.211975 & -2919.731459 \\
\hline Int6 & 0.355121 & -3246.603463 \\
\hline Int7 & 0.23865 & -2648.454548 \\
\hline Int8 & 0.342755 & -3171.331636 \\
\hline
\end{tabular}




\begin{tabular}{|c|c|c|}
\hline Int9 & 0.231358 & -963.1084617 \\
\hline Int10 & 0.450103 & -3694.257304 \\
\hline Int11 & 0.098817 & -2208.962291 \\
\hline Int12 & 0.132753 & -365.3504504 \\
\hline Int13 & 0.265914 & -2838.22041667 \\
\hline Int14 & 0.144016 & -441.374936 \\
\hline Int15 & 0.286998 & -2839.397756 \\
\hline Int16 & 0.288142 & -2839.397732 \\
\hline Int17 & 0.265259 & -2762.961706 \\
\hline Int18 & 0.240478 & -963.5172469 \\
\hline Int19 & 0.316782 & -3442.607207 \\
\hline Int20 & 0.203098 & -1234.401383 \\
\hline TS1 & 0.173674 & -706.3761571 \\
\hline $\mathrm{TS} 2$ & 0.14102 & -516.5538207 \\
\hline $\mathrm{TS} 3$ & 0.172725 & -706.3634241 \\
\hline TS4 & 0.225574 & -2920.83589 \\
\hline TS5 & 0.109403 & -712.5579767 \\
\hline TS6 & 0.222934 & -2920.867282 \\
\hline TS7 & 0.225457 & -2920.859639 \\
\hline TS8 & 0.352212 & -3246.594915 \\
\hline TS9 & 0.346055 & -3246.54713 \\
\hline TS10 & 0.380329 & -3436.360674 \\
\hline
\end{tabular}




\begin{tabular}{|c|c|c|}
\hline TS11 & 0.340038 & -3171.303851 \\
\hline TS12 & 0.345434 & -3171.321758 \\
\hline TS13 & 0.453807 & -3694.23488 \\
\hline TS14 & 0.375328 & -3361.063997 \\
\hline TS15 & 0.37153 & -3285.823029 \\
\hline TS16 & 0.359031 & -3172.48929 \\
\hline TS17 & 0.312197 & -3442.606074 \\
\hline TS18 & 0.318792 & -59428 \\
\hline
\end{tabular}




\begin{tabular}{|c|c|c|c|c|c|}
\hline \multicolumn{6}{|c|}{ Cartesian Coordinates of Intermediates and Transition States } \\
\hline \multicolumn{3}{|l|}{$\mathrm{B}\left(\mathrm{C}_{6} \mathrm{~F}_{5}\right)_{3}$} & \multicolumn{3}{|l|}{$2 a$} \\
\hline B -0.00069400 & 0.00047300 & 0.00035700 & $\mathrm{~N}-1.79100700$ & -0.60769400 & 0.14567000 \\
\hline C 1.09581400 & -1.12271200 & -0.00006200 & H - 2.00328800 & -1.54418700 & -0.17357100 \\
\hline C $\quad 0.94604600$ & -2.31861200 & 0.71805300 & C -0.44540200 & -0.27344400 & 0.05870200 \\
\hline C 1.90997200 & -3.31872600 & 0.73839400 & C $\quad 0.52311100$ & -1.29913100 & 0.00548200 \\
\hline C 3.07893900 & -3.15315700 & 0.00098500 & C -0.00013400 & 1.06281800 & 0.05447800 \\
\hline C 3.27241000 & -1.98886200 & -0.73711600 & C 1.87921500 & -0.99643700 & -0.04145300 \\
\hline C $\quad 2.29506300$ & -1.00183900 & -0.71797200 & H $\quad 0.19472700$ & -2.33692400 & 0.00748500 \\
\hline C - 1.52130000 & -0.38843400 & -0.00008600 & C $\quad 1.36527500$ & 1.35135300 & 0.01060400 \\
\hline C - 2.01490700 & -1.49005200 & -0.71522200 & H - 0.71676900 & 1.87681000 & 0.08619900 \\
\hline C -3.35843600 & -1.84255600 & -0.73619800 & C 2.31692700 & 0.33278100 & -0.03858100 \\
\hline C -4.27147900 & -1.09051800 & -0.00238100 & H $\quad 2.60257900$ & -1.80764800 & -0.08163700 \\
\hline C - 3.83172400 & 0.00650400 & 0.73303500 & H 1.68243200 & 2.39181000 & 0.00880400 \\
\hline C - 2.48349500 & 0.34077300 & 0.71467100 & Н $\quad 3.37724700$ & 0.56602200 & -0.07552700 \\
\hline C $\quad 0.42405800$ & 1.51139800 & 0.00089800 & C -2.83943300 & 0.36435100 & -0.10293000 \\
\hline C 1.54159800 & 1.97763000 & 0.71007300 & H - 2.75455900 & 0.85957600 & -1.08415100 \\
\hline C 1.92736600 & 3.31202700 & 0.72917500 & H -3.80442400 & -0.14695400 & -0.05532800 \\
\hline C 1.19368600 & 4.24375600 & 0.00029400 & H - 2.83824800 & 1.14161800 & 0.67021300 \\
\hline C $\quad 0.08157700$ & 3.83165500 & -0.72839500 & & & \\
\hline C -0.28604000 & 2.49210500 & -0.70832200 & $1 \mathrm{a}-\mathrm{B}$ & & \\
\hline F - 0.15710900 & -2.53163000 & 1.45624200 & C $\quad 0.22113000$ & -0.94342000 & 2.94701600 \\
\hline F $\quad 1.72565100$ & -4.43387400 & 1.45502400 & O $\quad 0.19424200$ & 0.03718600 & 2.19188800 \\
\hline F $\quad 4.01006200$ & -4.10688100 & 0.00158700 & O $\quad 0.32483000$ & -0.80677400 & 4.23752600 \\
\hline F $\quad 4.39184600$ & -1.83121300 & -1.45341900 & H $\quad 0.17618100$ & -1.97957900 & 2.61802800 \\
\hline F $\quad 2.53374800$ & 0.09544700 & -1.45709400 & H $\quad 0.35029900$ & 0.14684800 & 4.47623400 \\
\hline F - 1.18308600 & -2.24848100 & -1.45011400 & В 0.10682600 & 0.06410100 & 0.56785700 \\
\hline F - 3.78014700 & -2.89313200 & -1.44997500 & C $\quad 1.64590500$ & 0.47063100 & 0.21466200 \\
\hline F -5.56290400 & -1.42022600 & -0.00353000 & C 2.07667600 & 1.65626100 & -0.38454000 \\
\hline F -4.70656800 & 0.72543300 & 1.44638400 & C 3.41998200 & 1.94983600 & -0.62056300 \\
\hline F -2.11753700 & 1.40403900 & 1.45138600 & C 4.40285300 & 1.04634600 & -0.23950300 \\
\hline F - 1.36331700 & 2.15376200 & -1.43782800 & C 4.02868100 & -0.14314400 & 0.37762400 \\
\hline F - 0.62049400 & 4.72454500 & -1.43606900 & C $\quad 2.67959100$ & -0.39518200 & 0.58551200 \\
\hline F $\quad 1.55495700$ & 5.52668700 & 0.00024700 & C - 1.04961600 & 1.15493200 & 0.21350300 \\
\hline F $\quad 2.99210400$ & 3.70780900 & 1.43679600 & C - 1.90827500 & 1.80028900 & 1.10384200 \\
\hline \multirow[t]{2}{*}{ F $\quad 2.28374800$} & 1.12720000 & 1.44028100 & C -2.92845300 & 2.65822900 & 0.69184400 \\
\hline & & & C -3.13113000 & 2.89096500 & -0.66142800 \\
\hline $1 \mathrm{a}$ & & & C -2.30828300 & 2.25994800 & -1.58964900 \\
\hline C - 0.13089800 & 0.40076000 & 0.00002700 & C -1.30554000 & 1.41386400 & -1.13621800 \\
\hline O -1.13934800 & -0.26268900 & -0.00001200 & C -0.39821300 & -1.39798500 & 0.03488700 \\
\hline O $\quad 1.11886300$ & -0.09180500 & -0.00000700 & C $\quad 0.15190900$ & -2.08188900 & -1.05299600 \\
\hline Н -0.09490300 & 1.49973500 & 0.00003100 & C -0.37897800 & -3.26730800 & -1.55814700 \\
\hline \multirow[t]{2}{*}{ H $\quad 1.04417300$} & -1.06834200 & -0.00004100 & C -1.51385400 & -3.81936400 & -0.97583500 \\
\hline & & & C - 2.10601800 & -3.17461300 & 0.10483200 \\
\hline
\end{tabular}




\begin{tabular}{|c|c|c|c|c|c|c|}
\hline \multicolumn{2}{|c|}{ C - 1.54795700} & \multirow{2}{*}{$\begin{array}{r}-1.98887000 \\
2.60877000\end{array}$} & \multirow{2}{*}{$\begin{array}{l}0.56835500 \\
-0.76530800\end{array}$} & \multirow{2}{*}{$\begin{array}{l}\text { F - } 1.48577000 \\
\text { F - } 0.05181700\end{array}$} & \multirow{2}{*}{$\begin{array}{l}4.45332200 \\
2.38481500\end{array}$} & \multirow{2}{*}{$\begin{array}{l}-2.94056900 \\
-4.00986300\end{array}$} \\
\hline $\mathrm{F}$ & 1.20407400 & & & & & \\
\hline $\mathrm{F}$ & 3.76784800 & 3.10549000 & -1.20410600 & F $\quad 0.63932200$ & 0.30457000 & -2.54352100 \\
\hline $\mathrm{F}$ & 5.69430400 & 1.31853000 & -0.45438100 & F - 2.21625800 & -0.03206100 & 2.61925700 \\
\hline $\mathrm{F}$ & 4.96306400 & -1.02409700 & 0.76353100 & F - 4.60823700 & -1.13210800 & 2.40541000 \\
\hline $\mathrm{F}$ & 2.37625200 & -1.56631800 & 1.20345800 & F -5.31456200 & -2.56415400 & 0.17621300 \\
\hline & -1.80362500 & 1.62527600 & 2.44042300 & F -3.50505500 & -2.84763400 & -1.85997800 \\
\hline & -3.71905100 & 3.25437400 & 1.59828200 & F - 1.08680000 & -1.73978100 & -1.68842000 \\
\hline & -4.10584000 & 3.71240500 & -1.07018600 & $\mathrm{~N} \quad 0.40179000$ & 0.40447200 & 1.98319900 \\
\hline & -2.49141500 & 2.47114900 & -2.90133600 & H - 0.44278600 & 0.88175400 & 2.30150300 \\
\hline & -0.53237400 & 0.82420600 & -2.07494100 & C 1.49845600 & 1.39053300 & 2.14268200 \\
\hline & -2.17122500 & -1.39966700 & 1.62173400 & C 1.17471400 & 2.72437100 & 2.40110300 \\
\hline & -3.20029200 & -3.69177200 & 0.68087900 & C $\quad 2.83548000$ & 0.99027000 & 2.09951300 \\
\hline & -2.02993100 & -4.95910500 & -1.44682300 & C 2.19150200 & 3.66110100 & 2.59164400 \\
\hline 1 & 0.19574400 & -3.87789700 & -2.60333800 & H $\quad 0.13870900$ & 3.03795800 & 2.45620200 \\
\hline \multirow[t]{2}{*}{$F$} & 1.23961800 & -1.60892300 & -1.68878200 & C 3.84476200 & 1.93394800 & 2.29140700 \\
\hline & & & & H 3.10329600 & -0.04357700 & 1.91953100 \\
\hline \multicolumn{2}{|c|}{$2 a-B$} & & & C $\quad 3.52893900$ & 3.27084000 & 2.53505500 \\
\hline \multicolumn{2}{|c|}{ B -0.00860600} & -0.10582900 & 0.41696100 & H 1.92912000 & 4.69609200 & 2.79107200 \\
\hline $\mathrm{C}$ & 1.22205300 & -1.08468700 & -0.05553800 & H $\quad 4.88236500$ & 1.61543800 & 2.25138000 \\
\hline$C$ & 2.39685000 & -0.60512500 & -0.64767800 & H 4.31915800 & 4.00065100 & 2.68543900 \\
\hline$c$ & 3.45329200 & -1.41504000 & -1.05400700 & C $\quad 0.58904400$ & -0.74414500 & 2.93638600 \\
\hline $\mathrm{C}$ & 3.37773800 & -2.78883200 & 800 & $\mathrm{H}-0.23$ & -1.44347700 & 5600 \\
\hline$C$ & 2.24713200 & -3.32039100 & -0.25411000 & H $\quad 1.51974000$ & -1.25930300 & 2.71103800 \\
\hline$C$ & 1.21388100 & -2.47130000 & 0.13520800 & H $\quad 0.62402600$ & -0.35391000 & 3.95552800 \\
\hline & -0.31020000 & 1.20864700 & -0.53802100 & & & \\
\hline & -1.09903700 & 2.26712500 & -0.07808400 & TS6 & & \\
\hline & -1.48764600 & 3.36342300 & -0.83793400 & B -0.86820900 & -0.28504400 & -0.33954400 \\
\hline & -1.12259300 & 3.41661800 & -2.17704200 & C -0.05092700 & -1.23905400 & -1.33416500 \\
\hline & -0.38719900 & 2.36712900 & -2.71177600 & C $\quad 0.94450400$ & -0.71791000 & -2.16648300 \\
\hline & -0.01672600 & 1.29216500 & -1.90529500 & C 1.71852400 & -1.48388600 & -3.02688100 \\
\hline & -1.48652900 & -0.85597700 & 0.48391500 & C 1.48719600 & -2.85397600 & -3.10191300 \\
\hline & -1.91677000 & -1.58059900 & -0.63536800 & C $\quad 0.49728700$ & -3.42323800 & -2.31002400 \\
\hline & -3.17386300 & -2.16041500 & -0.75749300 & C -0.24472700 & -2.61888600 & -1.44770600 \\
\hline & -4.09770600 & -2.01583500 & 0.27214900 & C -1.53685700 & 1.03994500 & -0.95128200 \\
\hline & -3.73604200 & -1.29171500 & 1.39883500 & C -1.80158700 & 2.15419600 & -0.15052200 \\
\hline & -2.46307300 & -0.72934100 & 1.47289100 & C -2.38003900 & 3.32686100 & -0.61857100 \\
\hline $\mathrm{F}$ & 2.57339400 & 0.71727900 & -0.84558500 & C -2.74958200 & 3.40925900 & -1.95750600 \\
\hline$F$ & 4.54518000 & -0.87898300 & -1.62037200 & C - -2.52365500 & 2.32387700 & -2.79562400 \\
\hline $\mathrm{F}$ & 4.38456700 & -3.58466000 & -1.23755600 & C - 1.92871900 & 1.17202300 & -2.28618200 \\
\hline $\mathrm{F}$ & 2.16147000 & -4.64168200 & -0.03652000 & C -1.61265800 & -0.95862000 & 0.91180200 \\
\hline & 0.17230700 & -3.07975100 & 0.74676600 & C -2.98990600 & -0.86369300 & 1.13090600 \\
\hline & .56718300 & 2.25451000 & 1.20396800 & C - 3.63317200 & -1.46048100 & 2.21279500 \\
\hline & -2.22556200 & 4.34516300 & -0.29837900 & C -2.89244300 & -2.19008400 & 3.13480000 \\
\hline
\end{tabular}




\begin{tabular}{|c|c|c|c|c|c|c|}
\hline \multicolumn{2}{|c|}{ C - 1.51770500} & -2.31696300 & 2.95986100 & C $\quad 1.57637400$ & -2.69352000 & -2.51783900 \\
\hline \multicolumn{2}{|c|}{ C -0.91969700} & -1.71665400 & 1.85955600 & C $\quad 0.65690500$ & -2.21384400 & -1.58543900 \\
\hline $\mathrm{F}$ & 1.20608700 & 0.60945600 & -2.13639100 & C -1.56231000 & 0.87690800 & -0.75024900 \\
\hline $\mathrm{F}$ & 2.67302400 & -0.91989600 & -3.78012000 & C -2.16771500 & 1.78352400 & 0.12128300 \\
\hline $\mathrm{F}$ & 2.21081900 & -3.61542700 & -3.92860100 & C -3.07241700 & 2.76305400 & -0.27141300 \\
\hline $\mathrm{F}$ & 0.26194300 & -4.74095700 & -2.38215500 & C -3.43258700 & 2.85062400 & -1.61133200 \\
\hline \multicolumn{2}{|c|}{ F -1.19189500 } & -3.24626900 & -0.72401200 & C -2.87392700 & 1.96216800 & -2.52222000 \\
\hline \multicolumn{2}{|c|}{ F - 1.46513800} & 2.12635600 & 1.16114700 & C - 1.96602900 & 1.00149100 & -2.08162200 \\
\hline \multicolumn{2}{|c|}{ F - 2.58934300} & 4.36666400 & 0.20163500 & C -1.18377400 & -1.29884200 & 0.84018800 \\
\hline \multicolumn{2}{|c|}{ F -3.31545900 } & 4.52313600 & -2.43316600 & C -2.51595600 & -1.71277600 & 0.77154800 \\
\hline \multicolumn{2}{|c|}{$F-2.88346500$} & 2.39158400 & -4.08481600 & C -3.08599500 & -2.61943100 & 1.66232300 \\
\hline \multicolumn{2}{|c|}{ F - 1.75977900} & 0.15798200 & -3.15675700 & C -2.30913200 & -3.16472200 & 2.67719700 \\
\hline \multicolumn{2}{|c|}{ F $\quad 0.41718800$} & -1.87910600 & 1.73237000 & C -0.97218100 & -2.79737300 & 2.78018000 \\
\hline \multicolumn{2}{|c|}{ F - 0.79113500} & -3.01837900 & 3.84138300 & C -0.44525200 & -1.89522700 & 1.86300400 \\
\hline \multicolumn{2}{|c|}{ F -3.49435500 } & -2.76626500 & 4.18021000 & F $\quad 1.25513200$ & 1.33139700 & -1.82141200 \\
\hline \multicolumn{2}{|c|}{ F -4.95920600 } & -1.34000800 & 2.36710000 & F $\quad 3.05287500$ & 0.43801500 & -3.60073800 \\
\hline \multicolumn{2}{|c|}{ F -3.77879900 } & -0.18928600 & 0.27154800 & F $\quad 3.28211300$ & -2.24763100 & -4.09867100 \\
\hline \multicolumn{2}{|c|}{ Si 1.46820400} & 1.10133300 & 1.11899000 & F $\quad 1.67873000$ & -4.00995000 & -2.75778200 \\
\hline \multicolumn{2}{|c|}{ Н 1.35496600} & 2.42041100 & 0.46647500 & F -0.10001800 & -3.14888300 & -0.97547000 \\
\hline \multicolumn{2}{|c|}{ H $\quad 0.98357300$} & 1.01423000 & 2.50937800 & F - 1.86810800 & 1.74068900 & 1.44476400 \\
\hline \multicolumn{2}{|c|}{ H $\quad 0.31618600$} & 0.33152500 & 0.36967400 & F -3.60214100 & 3.61101300 & 0.62490100 \\
\hline \multicolumn{2}{|c|}{ C $\quad 3.04426000$} & 0.17635400 & 0.77496100 & F -4.30590900 & 3.78054700 & -2.01823600 \\
\hline \multicolumn{2}{|c|}{ C $\quad 3.83357200$} & 0.47952400 & -0.35065900 & F -3.21924700 & 2.03085500 & -3.81685300 \\
\hline \multicolumn{2}{|c|}{ C $\quad 3.47710100$} & -0.84246300 & 1.64434700 & F - 1.49420600 & 0.16334100 & -3.02708700 \\
\hline \multicolumn{2}{|r|}{ C $\quad 5.01671500$} & -0.21637200 & -0.59967400 & F $\quad 0.86906000$ & -1.59776600 & 2.00277300 \\
\hline \multicolumn{2}{|c|}{ H 3.52495900} & 1.25929200 & -1.04146700 & F - 0.20496200 & -3.32035200 & 3.74984700 \\
\hline \multicolumn{2}{|c|}{ C $\quad 4.66087800$} & -1.53688200 & 1.39553600 & F - 2.84058400 & -4.03619000 & 3.54410000 \\
\hline \multicolumn{2}{|c|}{ H $\quad 2.88866500$} & -1.09788500 & 2.52158700 & F - 4.37443000 & -2.97546200 & 1.54355200 \\
\hline $\mathrm{C}$ & 5.43100600 & -1.22505100 & 0.27292500 & F -3.32907500 & -1.25064300 & -0.20163100 \\
\hline $\mathrm{H}$ & 5.61417300 & 0.02858000 & -1.47376000 & Si 1.15813900 & 1.54465800 & 1.56395100 \\
\hline$\Pi$ & 4.98189300 & -2.32022900 & 2.07694700 & H $\quad 0.68355400$ & 2.67629900 & 0.74498000 \\
\hline $\mathrm{H}$ & 6.35287500 & -1.76713700 & 0.07858600 & H $\quad 0.44282700$ & 1.07546700 & 2.75779300 \\
\hline$C$ & 2.68409000 & 4.02124100 & 2.80254800 & H $\quad 0.32006600$ & 0.46247700 & 0.56901400 \\
\hline $\mathrm{O}$ & 3.07848600 & 2.89217100 & 2.60108700 & C 2.90033700 & 0.94620600 & 1.29449200 \\
\hline $\mathrm{H}$ & 1.72501700 & 4.42669100 & 2.45494200 & C 3.67251200 & 1.40734700 & 0.21212600 \\
\hline 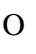 & 3.35845900 & 4.95019400 & 3.48312700 & C $\quad 3.48978500$ & 0.05059800 & 2.20624600 \\
\hline $\mathrm{H}$ & 4.20488100 & 4.55175000 & 3.77514000 & C 4.99261700 & 0.98592800 & 0.04725500 \\
\hline & & & & H $\quad 3.24233700$ & 2.08706200 & -0.51772400 \\
\hline Int & 3 & & & C 4.80971300 & -0.36929000 & 2.04091900 \\
\hline & -0.52456200 & -0.24446100 & -0.19913000 & H $\quad 2.91680300$ & -0.32952500 & 3.04767300 \\
\hline$c$ & 0.50063000 & -0.85786700 & -1.29175900 & C $\quad 5.56276300$ & 0.09811100 & 0.96177200 \\
\hline$C$ & 1.33483600 & -0.00813400 & -2.02162100 & H $\quad 5.57305800$ & 1.34771800 & -0.79724300 \\
\hline $\mathrm{C}$ & 2.27094900 & -0.43930800 & -2.95124700 & H $\quad 5.24836800$ & -1.06336300 & 2.75280100 \\
\hline $\mathrm{C}$ & 2.39075300 & -1.80202300 & -3.20425500 & H $\quad 6.59047800$ & -0.23095300 & 0.83232300 \\
\hline
\end{tabular}




\begin{tabular}{|c|c|c|c|c|c|c|}
\hline $\mathrm{C}$ & 1.74741400 & 4.29805900 & 2.72543100 & C 1.03970800 & 1.72850500 & -0.62174700 \\
\hline $\mathrm{O}$ & 1.87010100 & 3.09416100 & 2.93149900 & F - 2.73710300 & -0.59927100 & 2.11302900 \\
\hline $\mathrm{H}$ & 1.26487700 & 4.72503900 & 1.84060400 & F -5.05405200 & 0.57361000 & 2.80967400 \\
\hline $\mathrm{O}$ & 2.18220300 & 5.22568000 & 3.54933500 & F -6.02424500 & 2.67363500 & 1.34656100 \\
\hline \multirow[t]{2}{*}{$\mathrm{H}$} & 2.60510500 & 4.79732600 & 4.32512200 & F - 4.62260100 & 3.57362900 & -0.82045700 \\
\hline & & & & F - 2.32697300 & 2.42997400 & -1.52712400 \\
\hline \multicolumn{2}{|c|}{ TS7 } & & & F $\quad 1.22883700$ & -1.70276000 & 0.14162400 \\
\hline \multicolumn{2}{|c|}{ Si 3.58215900} & 0.21166200 & 3.39092500 & F $\quad 1.23308100$ & -4.33204500 & -0.39520600 \\
\hline $\mathrm{H}$ & 3.89598600 & -0.84538600 & 4.38206800 & F - 1.06017400 & -5.57153300 & -1.23694100 \\
\hline $\mathrm{H}$ & 3.45937400 & 1.54838500 & 4.00674900 & F - 3.35470800 & -4.11622800 & -1.52517900 \\
\hline $\mathrm{C}$ & 4.71263400 & 0.15354400 & 1.92837600 & F - 3.38344900 & -1.50284200 & -0.99622300 \\
\hline $\mathrm{C}$ & 5.68684500 & -0.85640300 & 1.81554300 & F $\quad 1.21111200$ & 1.97607000 & 0.69824800 \\
\hline $\mathrm{C}$ & 4.62306700 & 1.12391800 & 0.91109800 & F $\quad 2.91969000$ & 3.13528700 & -1.01046900 \\
\hline $\mathrm{C}$ & 6.54732700 & -0.89386200 & 0.71770000 & F $\quad 2.67654600$ & 2.73279400 & -3.70239400 \\
\hline 11 & 5.77783300 & -1.61749300 & 2.58722000 & F $\quad 0.67027500$ & 1.13521300 & -4.65945500 \\
\hline $\mathrm{C}$ & 5.47720200 & 1.08006300 & -0.18911500 & F - 1.05026600 & -0.03790500 & -2.96232400 \\
\hline $\mathrm{H}$ & 3.87738700 & 1.91118600 & 0.96720100 & H -0.36170000 & 0.30631500 & 1.09468500 \\
\hline $\mathrm{C}$ & 6.44104700 & 0.07257700 & -0.28451100 & & & \\
\hline $\mathrm{H}$ & 7.29703200 & -1.67687600 & 0.64338300 & $\mathrm{H}_{2}$ & & \\
\hline 11 & 5.38703900 & 1.83032200 & -0.96948700 & Н 0.00000000 & 0.00000000 & 0.37150900 \\
\hline $\mathrm{H}$ & 7.10961700 & 0.04129300 & -1.14082600 & Н 0.00000000 & 0.00000000 & -0.37150900 \\
\hline $\mathrm{C}$ & 1.16971700 & -1.09908200 & 3.18415900 & & & \\
\hline $\mathrm{O}$ & 1.95046600 & -0.10670800 & 2.86633300 & Int 4 & & \\
\hline $\mathrm{H}$ & 1.54028800 & -1.83193400 & 3.91314400 & C $\quad 3.27365100$ & -0.21321400 & 0.76762700 \\
\hline $\mathrm{O}$ & 0.03641400 & -1.23053000 & 2.71490900 & O $\quad 3.97170500$ & 0.08916000 & -0.17382200 \\
\hline & -0.26007100 & -0.32941100 & 1.68980900 & O 1.93478400 & -0.22653700 & 0.76053700 \\
\hline & -1.01873500 & 0.11457100 & -0.07566500 & H $\quad 3.66668300$ & -0.51502100 & 1.75047500 \\
\hline & -2.41078900 & 0.85732800 & 0.27059600 & Si 1.09043000 & 0.20074000 & -0.68607000 \\
\hline & -3.17364400 & 0.43674800 & 1.36358200 & H $\quad 1.47700300$ & -0.76425100 & -1.74163500 \\
\hline & -4.37374900 & 1.02332600 & 1.74426700 & H $\quad 1.43728800$ & 1.59348700 & -1.05105700 \\
\hline & -4.86929400 & 2.09128500 & 1.00266600 & C -0.72359700 & 0.07365400 & -0.28330800 \\
\hline & -4.15313000 & 2.54602300 & -0.09685500 & C - 1.38430800 & -1.16977500 & -0.27558000 \\
\hline & -2.95107000 & 1.92950500 & -0.44157700 & C - 1.47331500 & 1.22720800 & 0.01260300 \\
\hline & -1.08067800 & -1.47547300 & -0.37768300 & C -2.74256600 & -1.25821500 & 0.02847900 \\
\hline $\mathrm{C}$ & 0.06614600 & -2.26579200 & -0.26741000 & H - 0.83577700 & -2.07957100 & -0.51073000 \\
\hline 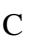 & 0.09850500 & -3.62888100 & -0.53773400 & C - 2.83330300 & 1.14208000 & 0.31458800 \\
\hline & -1.06408400 & -4.26098200 & -0.96507800 & H - -0.99333300 & 2.20339400 & 0.00521800 \\
\hline & -2.22982900 & -3.51779400 & -1.10647400 & C -3.46809500 & -0.10133400 & 0.32404400 \\
\hline & -2.21836500 & -2.15504500 & -0.81703700 & H -3.23623500 & -2.22649200 & 0.03122900 \\
\hline $\mathrm{C}$ & 0.01681400 & 0.88658900 & -1.05430500 & H -3.39679700 & 2.04387500 & 0.53943400 \\
\hline & -0.06823400 & 0.72486700 & -2.43881900 & H -4.52756100 & -0.16917700 & 0.55761400 \\
\hline C & 0.80253800 & 1.32910800 & -3.33899700 & & & \\
\hline $\mathrm{C}$ & 1.82071700 & 2.14525200 & -2.85672900 & Int5 & & \\
\hline $\mathrm{C}$ & 1.93528900 & 2.35019800 & -1.48785700 & C -1.63678800 & -0.41208800 & 0.97351900 \\
\hline
\end{tabular}




\begin{tabular}{|c|c|c|c|c|c|c|}
\hline & 89110100 & -0.27573100 & -0.01721500 & H -5.74418700 & -2.91706500 & 0.09021200 \\
\hline & 2.89726000 & -0.64467300 & 0.85426200 & C -7.62142000 & 0.48108800 & 0.12651900 \\
\hline & -1.27155900 & -0.33703200 & 1.99727200 & H -5.76287900 & 1.36794800 & -0.47625200 \\
\hline & 0.67415700 & 0.09191900 & -0.03690200 & C -8.29119300 & -0.69939700 & 0.45649800 \\
\hline C & 0.60937900 & 1.67154500 & -0.45152400 & H -8.13441200 & -2.83783800 & 0.69517000 \\
\hline $\mathrm{C}$ & 1.12067700 & 2.24559900 & -1.61756000 & H -8.15207100 & 1.42916300 & 0.13183400 \\
\hline C & 0.98916300 & 3.59974800 & -1.92666000 & \multirow[t]{2}{*}{ H -9.34517800} & -0.66921900 & 0.71980100 \\
\hline C & 0.31376100 & 4.44523300 & -1.05722300 & & & \\
\hline & -0.22585600 & 3.92240200 & 0.11348700 & \multicolumn{3}{|l|}{ TS8 } \\
\hline \multicolumn{2}{|c|}{ C -0.06589200} & 2.56961300 & 0.38092700 & $\mathrm{~N} \quad 1.78252100$ & 2.08448100 & -1.53254400 \\
\hline $\mathrm{C}$ & 1.35541100 & -0.91454800 & -1.12672600 & H $\quad 2.37780100$ & 1.49077800 & -2.11123300 \\
\hline C & 0.72772700 & -1.92232800 & -1.85952500 & C $\quad 2.58155700$ & 3.16211600 & -1.00280600 \\
\hline $\mathrm{C}$ & 1.41253800 & -2.78461800 & -2.71650000 & C 1.99612300 & 4.13667400 & -0.18555800 \\
\hline $\mathrm{C}$ & 2.78698800 & -2.66411600 & -2.86428900 & C 3.95445700 & 3.19785800 & -1.26572900 \\
\hline $\mathrm{C}$ & 3.46034000 & -1.67503000 & -2.15402100 & C 2.79107800 & 5.14998400 & 36900 \\
\hline $\mathrm{C}$ & 2.73929000 & -0.83864800 & -1.31236100 & H $\quad 0.93721100$ & 4.09912200 & 0.04761300 \\
\hline $\mathrm{C}$ & 1.31830400 & -0.24173300 & 1.43386800 & C $\quad 4.74072100$ & 4.21269400 & -0.72081600 \\
\hline C & 2.17891500 & 0.59792600 & 2.14546400 & H 4.40472300 & 2.43689200 & -1.89869200 \\
\hline $\mathrm{C}$ & 2.78871100 & 0.22999200 & 3.34340700 & C 4.16134600 & 5.19385100 & 0.08536600 \\
\hline $\mathrm{C}$ & 2.55416600 & -1.03069900 & 3.87914700 & H 2.33382800 & 5.90475400 & 0.98392300 \\
\hline $\mathrm{C}$ & 1.71188300 & -1.90939000 & 3.20656800 & H $\quad 5.80616900$ & 4.23531400 & -0.93152300 \\
\hline $\mathrm{C}$ & 1.13158500 & -1.50201700 & 2.01073600 & $\mathrm{H} \quad 4.77358400$ & 5.98551000 & 0.50751100 \\
\hline $\mathrm{F}$ & 1.77450900 & 1.50862800 & -2.53651 & C $\quad 0.60832600$ & 0 & 4000 \\
\hline $\mathrm{F}$ & 1.50468500 & 4.08775900 & -3.06440500 & H $\quad 0.93020900$ & 3.01296900 & -3.24089300 \\
\hline $\mathrm{F}$ & 0.17630600 & 5.74473400 & -1.34367400 & H $\quad 0.05852600$ & 1.58314000 & -2.61392400 \\
\hline & 0.88901200 & 4.72078700 & 0.96343100 & H -0.03781400 & 3.13766900 & -1.75634500 \\
\hline & -0.62180900 & 2.11891900 & 1.53586900 & C 1.42113200 & 0.75303400 & -0.07501700 \\
\hline & 0.60632200 & -2.13033900 & -1.78390600 & O $\quad 0.45120700$ & 0.00144900 & -0.46948000 \\
\hline $\mathrm{F}$ & 0.74767400 & -3.73348300 & -3.39520100 & O $\quad 2.63903400$ & 0.20870400 & -0.05039900 \\
\hline $\mathrm{F}$ & 3.45693500 & -3.48623400 & -3.68183200 & H $\quad 1.26885300$ & 1.52561600 & 0.66929100 \\
\hline $\mathrm{F}$ & 4.78825800 & -1.53950600 & -2.28832100 & B -1.03605900 & -0.27625100 & -0.02144700 \\
\hline $\mathrm{F}$ & 3.44036600 & 0.11338500 & -0.65682600 & C -1.32610700 & -1.61145500 & -0.94268500 \\
\hline $\mathrm{F}$ & 0.32018300 & -2.39992300 & 1.39632100 & C - 1.30547800 & -1.51857300 & -2.33828900 \\
\hline $\mathrm{F}$ & 1.47125800 & -3.12924200 & 3.70884400 & C - 1.44587700 & -2.59674000 & -3.20216800 \\
\hline $\mathrm{F}$ & 3.12910200 & -1.39411800 & 5.03062300 & C -1.61363600 & -3.87221400 & -2.67386400 \\
\hline $\mathrm{F}$ & 3.60375300 & 1.08206500 & 3.98 & C -1.63402200 & -4.02912200 & -1.29629200 \\
\hline $\mathrm{F}$ & 2.47913400 & 1.82852900 & 1.68957000 & C -1.48492200 & -2.91539400 & -0.46781600 \\
\hline & 3.77335000 & -0.82611900 & -0.67690000 & C -2.00144700 & 1.02842700 & -0.35104300 \\
\hline & 34028800 & -2.13452600 & -1.20263800 & C - 1.73626100 & 2.26825300 & 0.23301900 \\
\hline & 3.36242100 & 0.32137700 & -1.51023100 & C - -2.50197300 & 3.41449900 & 0.05295300 \\
\hline & 56663000 & -0.77840900 & -0.22737900 & C -3.64805000 & 3.34501100 & -0.72896400 \\
\hline & .25964700 & -1.95932800 & 0.10244200 & C - 3.98994100 & 2.12788700 & -1.30446000 \\
\hline & .27019200 & 0.44177900 & -0.21534200 & C -3.18183400 & 1.00974600 & -1.10166500 \\
\hline & -7.61108000 & -1.91943600 & 0.44373500 & C - 1.17839500 & -0.56206700 & 1.59590800 \\
\hline
\end{tabular}




\begin{tabular}{|c|c|c|c|c|c|}
\hline C - 2.46295300 & -0.78193000 & 2.10852300 & Н 3.70729400 & 2.80485100 & -3.16332300 \\
\hline C - 2.74420800 & -1.00791200 & 3.44991500 & C $\quad 4.16752800$ & 5.34200300 & -0.93829000 \\
\hline C - 1.70091000 & -1.02905200 & 4.36948000 & H 2.80631000 & 5.84772400 & 0.65823000 \\
\hline C -0.40467200 & -0.83084200 & 3.91719700 & Н $\quad 5.32452900$ & 4.59863600 & -2.60147200 \\
\hline C -0.17403800 & -0.61111600 & 2.55956800 & H $\quad 4.87597100$ & 6.12721500 & -0.69127200 \\
\hline F - 1.15880400 & -0.31009200 & -2.93498800 & C $\quad 0.04907600$ & 2.78422800 & -2.28752300 \\
\hline F - 1.42206500 & -2.41990700 & -4.53365500 & H $\quad 0.17144100$ & 3.44841200 & -3.14539300 \\
\hline F - 1.75011700 & -4.93023200 & -3.48385200 & H - 0.58239800 & 1.93662000 & -2.54326800 \\
\hline F - 1.78182700 & -5.25337900 & -0.76493400 & H - 0.37871200 & 3.33720700 & -1.45383600 \\
\hline F - 1.49025800 & -3.19223400 & 0.85368000 & C 1.37954400 & 1.09018100 & -0.73419300 \\
\hline F - 0.64825700 & 2.42239000 & 1.03674000 & O $\quad 0.33463600$ & 0.30333900 & -0.95404800 \\
\hline F - 2.13703100 & 4.57524400 & 0.62393300 & O $\quad 2.60299900$ & 0.49642100 & -0.94553800 \\
\hline F - 4.40885800 & 4.42915900 & -0.92170800 & H 1.38398400 & 1.66461800 & 0.18681200 \\
\hline F -5.10346600 & 2.03352700 & -2.04692600 & B -0.88451800 & -0.18757300 & -0.14352100 \\
\hline F -3.62841600 & -0.12444900 & -1.67428900 & C -1.37924200 & -1.38353900 & -1.17511400 \\
\hline F $\quad 1.13111200$ & -0.44432100 & 2.22397900 & C -1.88589300 & -1.06170100 & -2.43740900 \\
\hline F $\quad 0.61926600$ & -0.85299600 & 4.78591200 & C -2.22829400 & -1.99177100 & -3.41068500 \\
\hline F - 1.94413800 & -1.24340300 & 5.66905400 & C -2.05196900 & -3.34558800 & -3.14712800 \\
\hline F - 4.00449600 & -1.21414300 & 3.86261500 & C - 1.53169200 & -3.72658800 & -1.91920600 \\
\hline F -3.52001700 & -0.81752300 & 1.26578500 & C - 1.19870600 & -2.75345100 & -0.97570000 \\
\hline Si 2.98150400 & -1.39038500 & -0.58990400 & C -2.01070000 & 1.02322000 & 0.09341800 \\
\hline H $\quad 2.07390200$ & -2.33631700 & 0.09140700 & C - 1.66229000 & 2.20435200 & 0.75132600 \\
\hline H $\quad 2.80667900$ & -1.42470000 & -2.06439800 & C - -2.53586900 & 3.24427800 & 1.04965900 \\
\hline C $\quad 4.76354100$ & -1.69578700 & -0.15466800 & C -3.87712600 & 3.11493000 & 0.71399300 \\
\hline C $\quad 5.14010100$ & -2.03671500 & 1.15872400 & C -4.29761500 & 1.94756800 & 0.09060000 \\
\hline C $\quad 5.76912900$ & -1.60449300 & -1.13474400 & C -3.37860000 & 0.93742700 & -0.19235300 \\
\hline C $\quad 6.47687200$ & -2.26688200 & 1.48237900 & C -0.56958700 & -0.75102300 & 1.38524700 \\
\hline H $\quad 4.38541500$ & -2.12497900 & 1.93730900 & C -1.63137600 & -1.32586500 & 2.09626400 \\
\hline C $\quad 7.10734000$ & -1.83635900 & -0.81346600 & C -1.54253900 & -1.80403700 & 3.39747800 \\
\hline H $\quad 5.50713300$ & -1.35596200 & -2.16110800 & C -0.32907500 & -1.72244700 & 4.07279200 \\
\hline C $\quad 7.46145600$ & -2.16598700 & 0.49615900 & C $\quad 0.75938600$ & -1.16381400 & 3.42039000 \\
\hline H $\quad 6.75079300$ & -2.52818300 & 2.50106500 & C $\quad 0.61405200$ & -0.69379000 & 2.11570500 \\
\hline H $\quad 7.87120800$ & -1.76394200 & -1.58325900 & F - 2.08569600 & 0.23710200 & -2.77970700 \\
\hline H 8.50291100 & -2.34900100 & 0.74787300 & F - 2.72064900 & -1.59805200 & -4.59795600 \\
\hline & & & F - 2.37217900 & -4.26491900 & -4.06838500 \\
\hline Int6 & & & F - 1.33407100 & -5.02819200 & -1.65215500 \\
\hline $\mathrm{N} \quad 1.39847000$ & 2.26922200 & -1.90137500 & F - 0.65457700 & -3.23302400 & 0.16483400 \\
\hline H 1.77052000 & 1.75965400 & -2.70864700 & F -0.37397600 & 2.40780600 & 1.15111000 \\
\hline C $\quad 2.35480300$ & 3.33627100 & -1.56781800 & F - 2.09418400 & 4.35587200 & 1.66271100 \\
\hline C $\quad 2.08815900$ & 4.18247300 & -0.49026800 & F - 4.74725700 & 4.09527300 & 0.98851500 \\
\hline C 3.51253100 & 3.47503300 & -2.33012200 & F -5.59243100 & 1.79554400 & -0.22841200 \\
\hline C $\quad 3.00477300$ & 5.18731100 & -0.18059200 & F -3.90894100 & -0.16165400 & -0.76345900 \\
\hline H $\quad 1.18985600$ & 4.05772400 & 0.10584900 & F $\quad 1.74783800$ & -0.16698200 & 1.58050000 \\
\hline C $\quad 4.42036900$ & 4.48557000 & -2.01085100 & F $\quad 1.94707400$ & -1.07474800 & 4.04313800 \\
\hline
\end{tabular}




\begin{tabular}{|c|c|c|c|c|c|}
\hline F - 0.21555500 & -2.17696300 & 5.32810100 & C -0.76043400 & -3.44972400 & -3.55989400 \\
\hline F - 2.61064800 & -2.34497100 & 4.00486300 & C -0.11015800 & -3.84450000 & -2.40038900 \\
\hline F - 2.83660800 & -1.46126900 & 1.49812400 & C -0.10154400 & -3.00016200 & -1.28864300 \\
\hline Si 2.88961000 & -1.14625600 & -1.27040800 & C -2.12680200 & -0.02247300 & 0.43883100 \\
\hline H $\quad 1.87816500$ & -2.00205000 & -0.61568800 & C - -2.17221400 & 1.01478100 & 1.37336200 \\
\hline H 2.85527600 & -1.36244900 & -2.74332200 & C -3.33729600 & 1.58208900 & 1.87560600 \\
\hline C $\quad 4.61475000$ & -1.49577000 & -0.65192200 & C -4.56424800 & 1.08231400 & 1.45866200 \\
\hline C 4.93243000 & -1.39542000 & 0.71713200 & C -4.58610700 & 0.02471000 & 0.55956100 \\
\hline C $\quad 5.62575100$ & -1.89730100 & -1.54287400 & C -3.38899000 & -0.50725900 & 0.08227300 \\
\hline C $\quad 6.21814400$ & -1.68246300 & 1.17420100 & C -0.04009000 & -1.28096600 & 1.38218900 \\
\hline H $\quad 4.17132400$ & -1.09172600 & 1.43233200 & C -0.72704900 & -2.34604800 & 1.97908500 \\
\hline C $\quad 6.91298100$ & -2.18874500 & -1.08638000 & C -0.36539000 & -2.93980100 & 3.18171500 \\
\hline H 5.40877700 & -1.98527600 & -2.60547500 & C $\quad 0.74529400$ & -2.46366800 & 3.87223400 \\
\hline C $\quad 7.20977300$ & -2.08005000 & 0.27285100 & C 1.45823600 & -1.40276500 & 3.33505400 \\
\hline H $\quad 6.44714600$ & -1.60010500 & 2.23356000 & C 1.04431300 & -0.83725600 & 2.13040600 \\
\hline H $\quad 7.68133500$ & -2.49933000 & -1.78974500 & F - 1.97587800 & -0.20615500 & -2.57238100 \\
\hline H $\quad 8.21100600$ & -2.30627600 & 0.63080600 & F -2.00959700 & -1.79750300 & -4.70658400 \\
\hline & & & F - 0.77443700 & -4.24350700 & -4.63989500 \\
\hline TS10 & & & F $\quad 0.52187200$ & -5.02956200 & -2.35869200 \\
\hline $\mathrm{N} \quad 0.39480500$ & 2.67540200 & -1.39952500 & F $\quad 0.60461200$ & -3.46465400 & -0.23262200 \\
\hline H $\quad 1.38717100$ & 3.78552000 & -1.33972000 & F - 1.01428400 & 1.53138100 & 1.86557900 \\
\hline C -0.86080500 & 3.31235400 & -0.99354100 & F - 3.28625800 & 2.59809500 & 2.75314500 \\
\hline C - 2.05939700 & 3.10745800 & -1.68285100 & F -5.70678600 & 1.60939800 & 1.92035700 \\
\hline C -0.83065900 & 4.17361400 & 0.11003300 & F -5.76329500 & -0.48548000 & 0.16140100 \\
\hline C - 3.21489800 & 3.77842800 & -1.27566100 & F - 3.52923600 & -1.55707100 & -0.75307300 \\
\hline H -2.10667700 & 2.42865800 & -2.52550600 & F $\quad 1.80819200$ & 0.21031400 & 1.70998100 \\
\hline C - 1.99253900 & 4.82401200 & 0.52190000 & F $\quad 2.54239700$ & -0.92232100 & 3.97025500 \\
\hline H $\quad 0.09989800$ & 4.34324200 & 0.64455100 & F $\quad 1.11841300$ & -3.02294000 & 5.03107800 \\
\hline C -3.18777900 & 4.63490500 & -0.17515800 & F - 1.07186600 & -3.96571600 & 3.68161800 \\
\hline H - 4.14085500 & 3.61847700 & -1.82086000 & F - 1.80846900 & -2.86908800 & 1.35911500 \\
\hline H - 1.95831800 & 5.48746500 & 1.38117000 & Si 3.10181100 & -0.08069000 & -1.11397000 \\
\hline H -4.09061800 & 5.15073100 & 0.13906700 & H $\quad 2.37499400$ & -1.21727700 & -0.53383800 \\
\hline C $\quad 0.44335600$ & 2.25740400 & -2.82333000 & H 3.11274700 & -0.12971400 & -2.59367200 \\
\hline H $\quad 0.17902700$ & 3.11715400 & -3.44204300 & C $\quad 4.82892900$ & 0.11780600 & -0.45823700 \\
\hline H 1.46461200 & 1.95548500 & -3.06342300 & C $\quad 5.10837700$ & 0.04664700 & 0.92202000 \\
\hline H -0.23258500 & 1.42717400 & -3.03115800 & C $\quad 5.90068700$ & 0.29545100 & -1.35331900 \\
\hline C $\quad 0.76939300$ & 1.56695400 & -0.46581300 & C $\quad 6.41803100$ & 0.15779300 & 1.38603100 \\
\hline O $\quad 0.20332600$ & 0.40566000 & -0.74535800 & H $\quad 4.30348000$ & -0.09353200 & 1.63922600 \\
\hline O $\quad 2.26398500$ & 1.40946200 & -0.68763200 & C 7.21148800 & 0.40314200 & -0.88704200 \\
\hline H $\quad 0.67914200$ & 1.93095800 & 0.55418300 & H $\quad 5.71361300$ & 0.34622700 & -2.42372200 \\
\hline B - 0.65969300 & -0.65222600 & -0.02151700 & C $\quad 7.46967900$ & 0.33552500 & 0.48287300 \\
\hline C - 0.73500300 & -1.75710300 & -1.25120900 & H $\quad 6.61986000$ & 0.10228900 & 2.45231000 \\
\hline C -1.35530400 & -1.40660600 & -2.45495000 & H $\quad 8.02817200$ & 0.53805400 & -1.59109100 \\
\hline C -1.38692200 & -2.20849600 & -3.58845900 & H $\quad 8.49006300$ & 0.41812200 & 0.84797800 \\
\hline
\end{tabular}




\begin{tabular}{|c|c|c|c|c|c|c|}
\hline $\mathrm{C}$ & 3.15010100 & 4.48958600 & -0.71297500 & C -0.31262700 & -1.98565400 & 1.21816500 \\
\hline $\mathrm{O}$ & 2.04228600 & 4.66387500 & -1.34364200 & F $\quad 2.70055500$ & 2.02989200 & -1.09738300 \\
\hline $\mathrm{O}$ & 3.55252400 & 3.42853800 & -0.19360900 & F $\quad 2.60054000$ & 4.66945100 & -0.95879100 \\
\hline$\Pi$ & 3.77767800 & 5.38433500 & -0.64124400 & F $\quad 0.40763800$ & 5.94478600 & 0.06414200 \\
\hline \multirow[t]{2}{*}{$\mathrm{H}$} & 2.85030000 & 2.31867500 & -0.42512600 & F - 1.71648900 & 4.46102200 & 0.96078200 \\
\hline & & & & F - 1.64191500 & 1.79202000 & 0.85334400 \\
\hline \multicolumn{2}{|c|}{ Int7 } & & & F $\quad 0.77669300$ & -1.58705800 & -2.75156600 \\
\hline \multicolumn{2}{|c|}{$\mathrm{N}-2.80553000$} & -0.45226600 & -1.93749400 & F $\quad 2.99810700$ & -2.83037400 & -3.46977600 \\
\hline \multicolumn{2}{|c|}{ C -4.19828600} & -0.57134100 & -1.58512200 & F $\quad 5.27425100$ & -2.63867000 & -1.96554500 \\
\hline \multicolumn{2}{|c|}{ C -4.74441100} & 0.31801200 & -0.65394200 & F $\quad 5.25711000$ & -1.12630500 & 0.32024100 \\
\hline \multicolumn{2}{|c|}{ C -4.98928700} & -1.55803800 & -2.18057600 & F $\quad 3.02218700$ & 0.15924000 & 1.06985000 \\
\hline \multicolumn{2}{|c|}{ C -6.08952800} & 0.20145100 & -0.30201400 & F -0.50747300 & -2.61214500 & 0.03066900 \\
\hline \multicolumn{2}{|c|}{ H -4.12440000} & 1.09839800 & -0.22179800 & F - 0.96250900 & -4.01877800 & 2.26373200 \\
\hline \multicolumn{2}{|c|}{ C -6.33522400} & -1.65540300 & -1.83028000 & F -0.58684300 & -2.86777900 & 4.72310700 \\
\hline \multicolumn{2}{|c|}{ H -4.55983500 } & -2.25116300 & -2.89661900 & F $\quad 0.24869300$ & -0.27153000 & 4.88154300 \\
\hline \multicolumn{2}{|c|}{ C -6.88678600 } & -0.78096600 & -0.89061600 & F $\quad 0.70097300$ & 1.15063900 & 2.68332800 \\
\hline \multicolumn{2}{|c|}{ H -6.51343300} & 0.89072600 & 0.42230500 & & & \\
\hline \multicolumn{2}{|c|}{ H -6.95099500 } & -2.42388800 & -2.28832100 & $\mathrm{HOSiH} 2 \mathrm{Ph}$ & & \\
\hline \multicolumn{2}{|c|}{ H -7.93584400 } & -0.86353800 & -0.62165300 & O - 2.82434600 & -0.34366100 & 0.86948000 \\
\hline \multicolumn{2}{|c|}{ C -2.42623300} & -0.33867600 & -3.35534000 & Si-1.93137800 & 0.17134100 & -0.44811100 \\
\hline \multicolumn{2}{|c|}{ H -2.85629300} & 0.57476700 & -3.77537200 & H - 2.37134100 & -0.73149600 & -1.54022000 \\
\hline \multicolumn{2}{|c|}{ H -1.34127100 } & -0.31485500 & -3.43152100 & H - 2.24052700 & 1.58895200 & -0.79636000 \\
\hline \multicolumn{2}{|c|}{ H - 2.80566800} & -1.20369400 & -3.90275400 & C -0.07743100 & 0.06131700 & -0.20228200 \\
\hline \multicolumn{2}{|c|}{ C - 1.90420400} & -0.35494400 & -0.97553900 & C $\quad 0.58967300$ & -1.17927300 & -0.19046800 \\
\hline \multicolumn{2}{|c|}{ O - 0.68986600} & -0.09475100 & -1.22245700 & C $\quad 0.69133100$ & 1.22583800 & -0.02093000 \\
\hline \multicolumn{2}{|c|}{ H - 2.25569100} & -0.50293800 & 0.04260900 & C 1.96918100 & -1.25305200 & 0.00211400 \\
\hline \multicolumn{2}{|c|}{ B $\quad 0.46367800$} & 0.11428500 & -0.17979700 & H $\quad 0.02698400$ & -2.09932600 & -0.33678900 \\
\hline \multicolumn{2}{|c|}{ C $\quad 0.54349300$} & 1.75348100 & -0.09581200 & C $\quad 2.07260200$ & 1.15729500 & 0.17199100 \\
\hline \multicolumn{2}{|c|}{ C $\quad 1.58663900$} & 2.56070700 & -0.55365000 & H $\quad 0.20716400$ & 2.20036100 & -0.03466200 \\
\hline $\mathrm{C}$ & 1.56066900 & 3.95513300 & -0.50214200 & C $\quad 2.71221200$ & -0.08347600 & 0.18478500 \\
\hline $\mathrm{C}$ & 0.44957600 & 4.60773600 & 0.01349300 & H $\quad 2.46635800$ & -2.21977100 & 0.00659700 \\
\hline & -0.62594100 & 3.85211000 & 0.46849900 & H $\quad 2.64824600$ & 2.06916700 & 0.30955900 \\
\hline & -0.55088300 & 2.46783100 & 0.40020400 & H 3.78763900 & -0.14012300 & 0.33335100 \\
\hline $\mathrm{C}$ & 1.78584100 & -0.63190700 & -0.79232700 & H -2.63587600 & 0.11084800 & 1.70497600 \\
\hline $\mathrm{C}$ & 1.84845300 & -1.41872100 & -1.94305300 & & & \\
\hline $\mathrm{C}$ & 3.00269400 & -2.09050400 & -2.34818800 & $4 a$ & & \\
\hline $\mathrm{C}$ & 4.15955800 & -1.99866200 & -1.58751200 & $\mathrm{~N}-1.25457900$ & 0.19066700 & -0.10164700 \\
\hline $\mathrm{C}$ & 4.14725900 & -1.23041200 & -0.42748400 & C $\quad 0.16028700$ & 0.06173400 & -0.04493900 \\
\hline $\mathrm{C}$ & 2.97667700 & -0.57915700 & -0.06214700 & C $\quad 0.77790200$ & -1.16383300 & -0.34117200 \\
\hline $\mathrm{C}$ & 0.09656300 & -0.64846600 & 1.23269400 & C $\quad 0.95704900$ & 1.16707400 & 0.28876400 \\
\hline$C$ & 0.27915400 & -0.11740900 & 2.51205900 & C $\quad 2.16506000$ & -1.28516900 & -0.26501200 \\
\hline $\mathrm{C}$ & 0.05563800 & -0.84060800 & 3.68230100 & H $\quad 0.17752600$ & -2.01116000 & -0.65694800 \\
\hline & 0.36599500 & -2.16250200 & 3.60727800 & C 2.34486200 & 1.04001700 & 0.34063000 \\
\hline & -0.55630700 & -2.74326800 & 2.35857300 & H $\quad 0.49430500$ & 2.12120600 & 0.51942800 \\
\hline
\end{tabular}




\begin{tabular}{|c|c|c|c|c|c|c|}
\hline $\mathrm{C}$ & 2.95639900 & -0.18610600 & 0.07350800 & C $\quad 3.75272800$ & -0.73866700 & 0.30117400 \\
\hline 11 & 2.62806200 & -2.24091600 & -0.49566500 & H 2.88342100 & -2.71373900 & 0.33387400 \\
\hline $\mathrm{H}$ & 2.94822100 & 1.90586600 & 0.60031800 & H $\quad 4.31890900$ & 1.34017600 & 0.19599400 \\
\hline $\mathrm{H}$ & 4.03759300 & -0.28147400 & 0.11815700 & H 4.75359500 & -1.08366700 & 0.54487900 \\
\hline & -1.86118100 & 1.44238600 & -0.55465600 & C -0.32510400 & 1.55651700 & -1.71998600 \\
\hline & -1.29246100 & 1.84270800 & -1.39725200 & Н $\quad 0.12229300$ & 1.15809000 & -2.63433600 \\
\hline & -2.88302600 & 1.22560300 & -0.86720800 & H - 1.39224700 & 1.72359300 & -1.86608700 \\
\hline & -1.89433400 & 2.19044100 & 0.24665400 & H $\quad 0.15003000$ & 2.50621300 & -1.46476800 \\
\hline & -2.08114400 & -0.80244800 & 0.36022700 & & & \\
\hline & -3.30278100 & -0.75842500 & 0.34094600 & Int 2 & & \\
\hline \multirow{2}{*}{\multicolumn{2}{|c|}{ H - 1.52699300}} & -1.66146900 & 0.77238100 & N 0.89688100 & 0.48807000 & -0.09524500 \\
\hline & & & & C- 0.47409200 & 0.18521800 & -0.05317400 \\
\hline \multicolumn{2}{|c|}{ Int 1} & & & C-0.95422900 & -1.14153200 & -0.01917900 \\
\hline \multicolumn{2}{|c|}{ C -1.90891900 } & -0.15375500 & 0.00079000 & C-1.42470800 & 1.22793400 & -0.05636500 \\
\hline \multicolumn{2}{|c|}{ O - 1.20540900} & -1.15729000 & 0.00130800 & C-2.32386600 & -1.40276600 & -0.03571900 \\
\hline \multicolumn{2}{|c|}{ O - 1.49130800} & 1.09225500 & -0.00178500 & H-0.26518000 & -1.97371600 & 0.06122300 \\
\hline \multicolumn{2}{|c|}{ H -3.00480600 } & -0.21497800 & 0.00260200 & C-2.79035000 & 0.95123800 & -0.05833200 \\
\hline \multicolumn{2}{|c|}{ H -0.48652100 } & 1.12040500 & -0.00181600 & H-1.09634400 & 2.26156000 & -0.07268500 \\
\hline \multicolumn{2}{|c|}{ C 1.90920200} & 0.15329000 & 0.00096700 & C-3.25603500 & -0.36472100 & -0.05811600 \\
\hline \multicolumn{2}{|c|}{ O $\quad 1.20662600$} & 1.15748500 & 0.00095800 & H-2.66002300 & -2.43659000 & -0.00883300 \\
\hline \multicolumn{2}{|c|}{ O 1.49002700} & -1.09218600 & -0.00180400 & H-3.49561400 & 1.77899200 & -0.06576300 \\
\hline \multicolumn{2}{|c|}{ H 3.00518300} & 0.21339700 & 0.00350900 & H-4.32156600 & -0.57620700 & -0.06156000 \\
\hline \multirow[t]{2}{*}{$\mathrm{H}$} & 0.48495400 & -1.11813800 & -0.00425800 & C 1.36004600 & 1.73423200 & 0.51434800 \\
\hline & & & & Н 0.93831700 & 1.87894600 & 1.51781300 \\
\hline \multicolumn{2}{|c|}{ TS1 } & & & Н 2.44864200 & 1.70874100 & 0.58526100 \\
\hline \multicolumn{2}{|c|}{ C -3.00338000} & -1.60480100 & -0.22387600 & H 1.09630700 & 2.60625600 & -0.09650000 \\
\hline \multicolumn{2}{|c|}{ O -2.00300800} & -1.56371100 & -0.95044400 & C 1.86408300 & -0.57685800 & -0.29008300 \\
\hline \multicolumn{2}{|c|}{$\mathrm{O}-3.45235100$} & -0.67312800 & 0.55507600 & O 3.06047800 & -0.05504400 & -0.82561200 \\
\hline \multicolumn{2}{|c|}{ H -3.61403700 } & -2.52517800 & -0.19539800 & O 2.20989400 & -1.29720600 & 0.85817300 \\
\hline \multicolumn{2}{|c|}{ H -2.88104300 } & 0.36595200 & 0.45735700 & H 1.41827000 & -1.31114900 & -0.96714200 \\
\hline \multicolumn{2}{|c|}{ C - 1.01892100} & 1.23034700 & 0.82924200 & Н 2.67124700 & -0.68623200 & 1.45977400 \\
\hline \multicolumn{2}{|c|}{ O - 2.24376400} & 1.43304100 & 0.46533100 & Н 2.81970400 & 0.47444500 & -1.60564800 \\
\hline \multicolumn{2}{|c|}{ O - 0.75999100} & 0.30047900 & 1.80609800 & & & \\
\hline \multicolumn{2}{|c|}{ H -0.39538000 } & 2.11506800 & 0.99966300 & $\mathrm{TS} 2$ & & \\
\hline \multicolumn{2}{|c|}{ H - 1.52075100} & -0.30785900 & 1.84629000 & N 0.87285700 & 0.29555400 & 0.46460700 \\
\hline \multicolumn{2}{|c|}{ N - 0.16770700} & 0.58909400 & -0.60902700 & C- 0.52541800 & 0.10827800 & 0.23214100 \\
\hline & -0.77171100 & -0.23209600 & -0.81033600 & C-1.14659900 & -1.08589800 & 0.62334500 \\
\hline $\mathrm{C}$ & 1.18281100 & 0.15042100 & -0.33223200 & C-1.28741600 & 1.12073000 & -0.36436100 \\
\hline $\mathrm{C}$ & 1.41853400 & -1.21274200 & -0.13015400 & C-2.50839900 & -1.27277700 & 0.39001600 \\
\hline $\mathrm{C}$ & 2.22660900 & 1.07263700 & -0.21110600 & H-0.57031300 & -1.85487200 & 1.12889600 \\
\hline$C$ & 2.70373500 & -1.65289900 & 0.18318100 & C-2.65303200 & 0.93107300 & -0.57818500 \\
\hline $\mathrm{H}$ & 0.59932100 & -1.91975000 & -0.22458300 & H-0.81701400 & 2.04929600 & -0.67106100 \\
\hline $\mathrm{C}$ & 3.50918400 & 0.62153500 & 0.10437300 & C-3.26802100 & -0.26638900 & -0.20993600 \\
\hline $\mathrm{H}$ & 2.05160400 & 2.13341800 & -0.35976700 & H-2.97869000 & -2.20299900 & 0.69676700 \\
\hline
\end{tabular}




\begin{tabular}{|c|c|c|c|c|c|}
\hline H-3.23386400 & 1.72267600 & -1.04379200 & Н 0.00000000 & 0.76046100 & -0.48171600 \\
\hline H-4.33086200 & -0.41132600 & -0.38148100 & Н 0.00000000 & -0.76046100 & -0.48171600 \\
\hline C 1.36313100 & 1.61956800 & 0.85733900 & & & \\
\hline Н 0.68416000 & 2.04681400 & 1.59845300 & Int8 & & \\
\hline Н 2.35482900 & 1.50084600 & 1.29180700 & B 1.44999800 & -0.29857500 & -0.04050400 \\
\hline H 1.43046400 & 2.30034500 & -0.00058500 & C 1.94317700 & 0.80113200 & -1.14411200 \\
\hline C 1.75791700 & -0.65022500 & 0.06665800 & C 1.06777600 & 1.21030100 & -2.15200200 \\
\hline O 2.47672300 & -0.47536700 & -1.72235000 & C 1.36013900 & 2.17617000 & -3.10609400 \\
\hline O 3.01831000 & -0.58120500 & 0.46897300 & C $\quad 2.61181900$ & 2.78118700 & -3.09356700 \\
\hline H 1.30961300 & -1.61166800 & -0.17773700 & C $\quad 3.53028000$ & 2.40490200 & -2.12298100 \\
\hline Н 3.29016900 & -0.53090600 & -0.62653200 & C 3.18641300 & 1.43443900 & -1.18172200 \\
\hline \multirow[t]{2}{*}{ Н 2.38563800} & 0.46169200 & -1.97078500 & C $\quad 0.98798100$ & -1.73193300 & -0.69156200 \\
\hline & & & C $\quad 0.10058100$ & -2.57320200 & -0.01946200 \\
\hline TS3 & & & C -0.34808900 & -3.79584700 & -0.50978900 \\
\hline N 0.12637000 & -0.67830000 & -0.45826600 & C $\quad 0.11879000$ & -4.24512400 & -1.73852200 \\
\hline C 1.46440500 & -0.21350900 & -0.25150800 & C 1.02063400 & -3.45841000 & -2.44492900 \\
\hline C 2.39358800 & -1.03911900 & 0.39903600 & C 1.43715200 & -2.24028400 & -1.91266700 \\
\hline C 1.86533500 & 1.05015800 & -0.70599900 & C $\quad 2.46015700$ & -0.51048900 & 1.22492600 \\
\hline C 3.69653800 & -0.59323000 & 0.61171700 & C $\quad 3.47467400$ & -1.47013100 & 1.23889400 \\
\hline Н 2.09873300 & -2.03519100 & 0.71589600 & C $\quad 4.32470700$ & -1.68444800 & 2.31999600 \\
\hline C 3.17858700 & 1.48083200 & -0.50515600 & C 4.19065200 & -0.90035100 & 3.46002900 \\
\hline H 1.15732300 & 1.69656700 & -1.21557200 & C $\quad 3.21185000$ & 0.08470200 & 3.49139000 \\
\hline C 4.09604000 & 0.66708800 & 0.15970100 & C $\quad 2.38686700$ & 0.26183000 & 2.38363100 \\
\hline H 4.40694500 & -1.24325000 & 1.11519000 & F - 0.17027000 & 0.65491800 & -2.22904200 \\
\hline Н 3.47850000 & 2.46131600 & -0.86504200 & F $\quad 0.44846100$ & 2.53115900 & -4.02994400 \\
\hline H 5.11581700 & 1.00652200 & 0.31737500 & F $\quad 2.92497700$ & 3.71642300 & -4.00322400 \\
\hline C-0.57113100 & -0.29684500 & -1.68775600 & F $\quad 4.74590800$ & 2.97774700 & -2.09947200 \\
\hline Н 0.14797900 & -0.30379200 & -2.50882300 & F $\quad 4.15089800$ & 1.13281200 & -0.28474200 \\
\hline H-1.34931000 & -1.03061400 & -1.90201700 & F -0.39076800 & -2.21058100 & 1.19459700 \\
\hline H-1.03416100 & 0.69563200 & -1.62216700 & F - 1.21951700 & -4.54290100 & 0.19283700 \\
\hline C-0.60231100 & -1.05421000 & 0.65231300 & F - -0.29573100 & -5.42037300 & -2.23581200 \\
\hline O-1.11585200 & 0.42431800 & 1.50678800 & F $\quad 1.48961000$ & -3.88485800 & -3.62982200 \\
\hline O-1.73777100 & -1.71289500 & 0.51927300 & F $\quad 2.33423000$ & -1.55554300 & -2.65380800 \\
\hline Н 0.01728800 & -1.42892000 & 1.46138200 & F $\quad 1.47697200$ & 1.26139700 & 2.48319300 \\
\hline H-2.46623800 & -1.19624100 & 0.01054700 & F $\quad 3.07458500$ & 0.85904900 & 4.58257700 \\
\hline H-0.38822700 & 1.06960600 & 1.43791000 & F $\quad 4.99884400$ & -1.08972700 & 4.51400900 \\
\hline C-3.78055900 & 0.78712300 & -0.31398300 & F $\quad 5.27670700$ & -2.63197200 & 2.27068300 \\
\hline O-3.64779300 & -0.41864400 & -0.61810200 & F $\quad 3.69056900$ & -2.24645600 & 0.15315800 \\
\hline O-3.02271700 & 1.49912800 & 0.42392900 & Si-1.45766700 & 0.68649700 & 1.03170000 \\
\hline H-4.65327100 & 1.32393600 & -0.72888300 & H - 1.75591400 & -0.13840100 & -0.15313800 \\
\hline \multirow[t]{2}{*}{ H-2.02746800 } & 0.89959400 & 0.98397700 & H -0.96369800 & 0.12551400 & 2.29272000 \\
\hline & & & H $\quad 0.40014200$ & 0.20181000 & 0.43775700 \\
\hline $\mathrm{H} 2 \mathrm{O}$ & & & C - 1.16497700 & 2.50216200 & 0.74625800 \\
\hline O 0.00000000 & 0.00000000 & 0.12042900 & C -1.37263400 & 3.08961300 & -0.51573700 \\
\hline
\end{tabular}




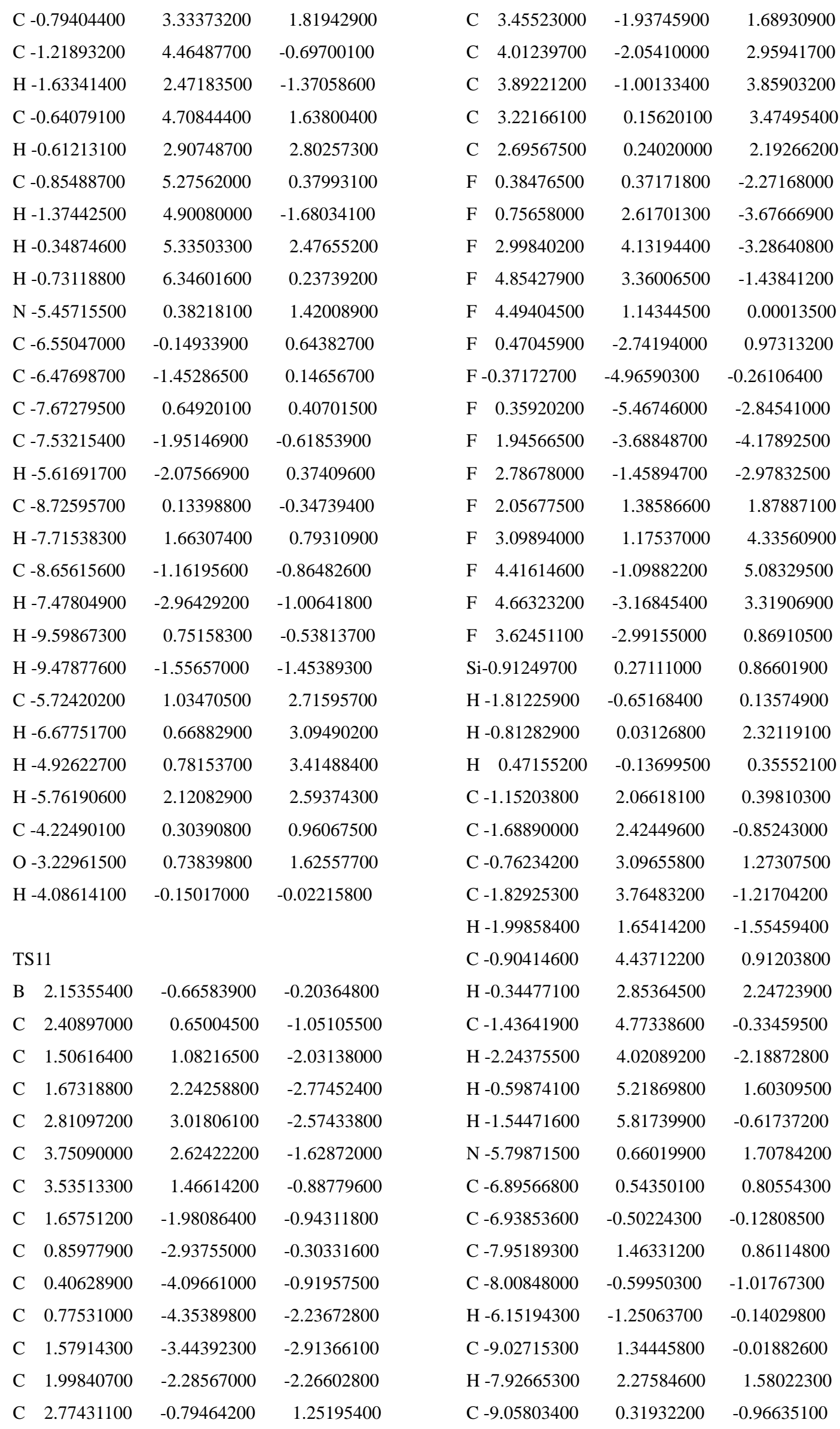




\begin{tabular}{|c|c|c|c|c|c|}
\hline H -8.02901200 & -1.41289800 & -1.73799500 & H $\quad 8.67388400$ & 0.28846000 & -0.53766400 \\
\hline H -9.83931700 & 2.06461800 & 0.03297600 & H $\quad 0.09928500$ & 0.52877500 & 0.17514800 \\
\hline H -9.89557900 & 0.23273300 & -1.65268200 & B -0.80296300 & -0.50034400 & -0.04162000 \\
\hline C -6.02785300 & 1.07405300 & 3.09391300 & C - 1.71778600 & 0.14747000 & -1.20705700 \\
\hline H -6.96371200 & 0.64067800 & 3.45288500 & C - -2.39725500 & 1.34622800 & -0.96638000 \\
\hline H -5.19655900 & 0.71086100 & 3.69887300 & C -3.18952600 & 1.99628000 & -1.90522600 \\
\hline H -6.07422000 & 2.16539100 & 3.18639400 & C -3.35342000 & 1.42606400 & -3.16324200 \\
\hline C -4.51635600 & 0.47346800 & 1.28525300 & C -2.72005900 & 0.22278800 & -3.44994000 \\
\hline $\mathrm{O}-3.52898700$ & 0.54928100 & 2.01265600 & C - 1.92718400 & -0.38897900 & -2.48104400 \\
\hline \multirow[t]{2}{*}{ H -4.43944900 } & 0.25285600 & 0.20986000 & C -1.56728900 & -0.67171300 & 1.38084600 \\
\hline & & & C -0.92162600 & -0.56401000 & 2.61136700 \\
\hline TS12 & & & C -1.54660000 & -0.71441700 & 3.84364200 \\
\hline $\mathrm{N} \quad 1.01461900$ & 2.58365500 & -0.32431100 & C - -2.90440500 & -1.01012800 & 3.88018500 \\
\hline C $\quad 0.18198500$ & 3.66758700 & 0.09002500 & C -3.59795600 & -1.15059200 & 2.68410800 \\
\hline C $\quad 0.16291900$ & 4.08313000 & 1.43024400 & C -2.92607700 & -0.98605300 & 1.47543700 \\
\hline C -0.59336500 & 4.35589300 & -0.85323900 & C $\quad 0.19330400$ & -1.70522000 & -0.44522100 \\
\hline C -0.64912700 & 5.14630400 & 1.82002100 & C $\quad 0.24912700$ & -2.94921200 & 0.19250600 \\
\hline H $\quad 0.79929200$ & 3.60215000 & 2.16616700 & C $\quad 1.17975400$ & -3.93719300 & -0.12738800 \\
\hline C - 1.39372900 & 5.42543800 & -0.45407000 & C 2.12134400 & -3.70011800 & -1.12039300 \\
\hline H - 0.58874700 & 4.05034500 & -1.89348400 & C 2.09655600 & -2.48566000 & -1.79854400 \\
\hline C -1.43238500 & 5.82232300 & 0.88288900 & C 1.14329900 & -1.53540100 & -1.45817800 \\
\hline H -0.65255800 & 5.45652400 & 2.86122400 & F - 2.28768000 & 1.94037900 & 0.24183800 \\
\hline H - 1.99700900 & 0100 & -1.1955 & F -3.79154400 & 3.16 & -1.6117 \\
\hline H -2.05972500 & 6.65385700 & 1.19030800 & F -4.11330700 & 2.02893000 & -4.08641000 \\
\hline C $\quad 1.56340100$ & 2.58734300 & -1.68469200 & F - 2.88075800 & -0.34315600 & -4.65648700 \\
\hline H $\quad 1.87764800$ & 3.60370200 & -1.93005200 & F - 1.37121500 & -1.56419200 & -2.83985100 \\
\hline H $\quad 2.42614600$ & 1.92542200 & -1.72119200 & F $\quad 0.41268300$ & -0.29448500 & 2.65581900 \\
\hline H $\quad 0.82556400$ & 2.24827400 & -2.41864700 & F - 0.85577800 & -0.58167500 & 4.98671700 \\
\hline C $\quad 1.16215500$ & 1.48410400 & 0.46910600 & F -3.53387000 & -1.16088600 & 5.05208700 \\
\hline O $\quad 2.26658400$ & 0.75413400 & 0.25902000 & F -4.90569600 & -1.44927600 & 2.70333100 \\
\hline H $\quad 0.84101400$ & 1.62053300 & 1.49719900 & F -3.66490900 & -1.16826900 & 0.36079200 \\
\hline Si 3.24848800 & -0.10446700 & 1.36512300 & F $\quad 1.15785300$ & -0.38955400 & -2.17376600 \\
\hline H 3.13145900 & 0.55721400 & 2.68715400 & F $\quad 3.01087300$ & -2.23198300 & -2.75297700 \\
\hline H $\quad 2.76602600$ & -1.50111900 & 1.41144800 & F $\quad 3.03091500$ & -4.63072100 & -1.43198200 \\
\hline C $\quad 4.99049000$ & 0.02574800 & 0.72306500 & F $\quad 1.17608900$ & -5.11140200 & 0.52166300 \\
\hline C $\quad 5.36472000$ & -0.50598500 & -0.52722700 & F - 0.62582800 & -3.27296000 & 1.16618700 \\
\hline C $\quad 5.97771300$ & 0.65427400 & 1.50447700 & & & \\
\hline C $\quad 6.68041500$ & -0.40754500 & -0.97803500 & Int9 & & \\
\hline H 4.63005200 & -0.99640900 & -1.16171600 & $\mathrm{~N}-1.97843900$ & -1.29453900 & 0.56702400 \\
\hline C $\quad 7.29666300$ & 0.74693800 & 1.05611600 & C - -2.50115300 & -0.02716300 & 0.27474300 \\
\hline H $\quad 5.71785100$ & 1.07517400 & 2.47329400 & C - 1.66892000 & 1.09520700 & 0.06787100 \\
\hline C 7.64793100 & 0.21691100 & -0.18607000 & C - 3.89617300 & 0.17067700 & 0.19443500 \\
\hline H $\quad 6.95280300$ & -0.82061000 & -1.94558200 & C - -2.21546100 & 2.35378700 & -0.17073000 \\
\hline H 8.04657600 & 1.23328300 & 1.67434300 & H -0.59069000 & 0.98871500 & 0.06483200 \\
\hline
\end{tabular}




\begin{tabular}{|c|c|c|c|c|c|}
\hline C -4.42742400 & 1.43627000 & -0.05164000 & C -3.21930600 & 1.19819900 & 2.28537100 \\
\hline H -4.57595400 & -0.66193300 & 0.33238900 & C -3.39597300 & 1.22778800 & 3.66689400 \\
\hline C -3.59770400 & 2.54275000 & -0.23105300 & C -2.87895500 & 0.20086300 & 4.44751200 \\
\hline H - 1.54356000 & 3.19457300 & -0.32621000 & C -2.19530600 & -0.84365200 & 3.83367800 \\
\hline H -5.50772200 & 1.55045000 & -0.10199400 & C -2.05276300 & -0.83703200 & 2.45172400 \\
\hline H -4.01624200 & 3.52635400 & -0.42426300 & F - 1.57698700 & -0.74631600 & -2.61873900 \\
\hline C - 2.82750900 & -2.46894900 & 0.40700500 & F - 2.65036000 & -2.78072400 & -4.00699200 \\
\hline H -3.26915400 & -2.52885900 & -0.59706500 & F - 4.72129200 & -4.22466300 & -2.94996100 \\
\hline H - 2.22355100 & -3.36460000 & 0.56502300 & F -5.68910300 & -3.59027000 & -0.47494800 \\
\hline H -3.64001300 & -2.48011600 & 1.14426400 & F -4.63469900 & -1.58227700 & 0.92461000 \\
\hline C -0.57068700 & -1.50270900 & 0.64333600 & F -0.42378400 & 2.23705800 & 0.62454300 \\
\hline O $\quad 0.02438400$ & -1.62064700 & -0.66261200 & F -0.27029600 & 4.64592100 & -0.53561100 \\
\hline H - 0.10467100 & -0.67513800 & 1.18754500 & F - 2.05143300 & 5.34945300 & -2.49737500 \\
\hline Si 1.66334000 & -1.79051300 & -0.92616100 & F -3.97884700 & 3.57356600 & -3.26982900 \\
\hline H 2.18361600 & -3.01366600 & -0.24536700 & F -4.14414600 & 1.16655200 & -2.13866900 \\
\hline H 1.78062500 & -1.93089600 & -2.39803800 & F - 1.39088900 & -1.88589700 & 1.90750100 \\
\hline C $\quad 2.67651900$ & -0.33594600 & -0.31405900 & F -1.69292200 & -1.84381400 & 4.57270700 \\
\hline C $\quad 2.65339800$ & 0.90255400 & -0.98590300 & F -3.03767000 & 0.21523100 & 5.77558600 \\
\hline C 3.48501700 & -0.44847800 & 0.83228200 & F -4.06495300 & 2.23475600 & 4.24668500 \\
\hline C 3.40241900 & 1.98620700 & -0.52627400 & F -3.76836600 & 2.21883800 & 1.59634900 \\
\hline H $\quad 2.04784200$ & 1.02325600 & -1.88 & Si 0.61089900 & -0.47667200 & -0.19157000 \\
\hline C $\quad 4.23766600$ & 0.63287800 & 1.29511700 & H $\quad 0.83308000$ & 0.55504700 & -1.21383400 \\
\hline H 3.53310800 & -1.39340700 & 1.37001000 & H $\quad 0.92636500$ & -0.19729500 & 1.21919900 \\
\hline C 4.19549400 & 1.85204000 & 0.61642200 & H -0.98100400 & -0.30454400 & -0.07328600 \\
\hline H 3.37204300 & 2.93270200 & -1.06001600 & C $\quad 0.86642700$ & -2.22976800 & -0.74989900 \\
\hline H $\quad 4.85603800$ & 0.52392700 & 2.18249700 & C 1.04531200 & -2.52363900 & -2.11545200 \\
\hline H $\quad 4.78146300$ & 2.69492600 & 0.97397900 & C $\quad 0.89926500$ & -3.28980700 & 0.17634000 \\
\hline \multirow[t]{2}{*}{ H -0.38883900} & -2.42691000 & 100 & C $\quad 1.25074700$ & -3.83634500 & -2.54023400 \\
\hline & & & H 1.02449400 & -1.72662500 & -2.85389200 \\
\hline TS13 & & & C 1.10575100 & -4.60202700 & -0.24991700 \\
\hline B -2.33475600 & 0.13411000 & 0.02294400 & H $\quad 0.75717000$ & -3.09468400 & 1.23562800 \\
\hline C -3.03508800 & -1.07517600 & -0.77414000 & C $\quad 1.28128000$ & -4.87614800 & -1.60807300 \\
\hline C - 2.59507000 & -1.43497800 & -2.05092400 & H 1.38655300 & -4.04720200 & -3.59753000 \\
\hline-3.12924200 & -2.48084200 & -2.79114500 & $\mathrm{H} \quad 1.12831900$ & -5.40900900 & 0.47752500 \\
\hline C -4.18261500 & -3.21603400 & -2.25597300 & $\mathrm{H} \quad 1.44067100$ & -5.89884500 & -1.93965100 \\
\hline C -4.66929300 & -2.89225500 & -0.99554700 & $\mathrm{~N} \quad 5.22818700$ & -0.34712800 & 1.18410500 \\
\hline C -4.09508200 & -1.84100700 & -0.28317700 & C $\quad 6.01179200$ & -1.44057900 & 0.76813500 \\
\hline C -2.27484000 & 1.56907200 & -0.71184400 & C $\quad 5.48522700$ & -2.40945300 & -0.11228100 \\
\hline C - 1.32824500 & 2.52832500 & -0.34546400 & C 7.34393300 & -1.59157000 & 1.20060700 \\
\hline C -1.22387700 & 3.78502200 & -0.92590900 & С $\quad 6.26016900$ & -3.49266900 & -0.52005400 \\
\hline C -2.12394200 & 4.14394500 & -1.92386800 & $\mathrm{H} \quad 4.47590300$ & -2.30972800 & -0.49552500 \\
\hline C -3.09933200 & 3.23507500 & -2.31665300 & C 8.11015600 & -2.67691600 & 0.77420100 \\
\hline C -3.15791300 & 1.98002900 & -1.71351900 & H $\quad 7.78832300$ & -0.87227600 & 1.87837900 \\
\hline C -2.53425600 & 0.17730900 & 1.62156100 & C $\quad 7.57854100$ & -3.63923100 & -0.08363700 \\
\hline
\end{tabular}




\begin{tabular}{|c|c|c|c|c|c|c|}
\hline $\mathrm{H}$ & 5.82510400 & -4.22319700 & -1.19755200 & C - 1.29009100 & 2.81365500 & 2.59950200 \\
\hline $\mathrm{H}$ & 9.13431500 & -2.76737600 & 1.12788400 & C -1.29654800 & 1.60762500 & 1.90226400 \\
\hline $\mathrm{H}$ & 8.17975800 & -4.48282900 & -0.41046500 & F - 1.25826500 & -2.90888400 & 0.00682200 \\
\hline $\mathrm{C}$ & 5.81040200 & 0.64133500 & 2.08796800 & F - 2.73089500 & -4.89254800 & 1.03305500 \\
\hline $\mathrm{H}$ & 6.71428700 & 1.07857400 & 1.65203100 & F -5.17644500 & -4.37212200 & 2.15031800 \\
\hline 11 & 5.09458100 & 1.45010400 & 2.24157500 & F -6.11511000 & -1.80252900 & 2.20992700 \\
\hline 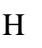 & 6.07034700 & 0.21244100 & 3.06709800 & F -4.66932000 & 0.19703300 & 1.18809200 \\
\hline $\mathrm{C}$ & 3.79975300 & -0.44407500 & 1.12696200 & F $\quad 0.33924300$ & 0.21734600 & -1.94009100 \\
\hline $\mathrm{O}$ & 3.22943500 & -0.08283500 & -0.15614100 & F $\quad 0.19491900$ & 0.37746000 & -4.59412100 \\
\hline $\mathrm{H}$ & 3.46304300 & -1.46830600 & 1.31350300 & F - -2.23194800 & 0.27680300 & -5.86964300 \\
\hline & 3.98430200 & 0.98824700 & -1.22964200 & F - 4.52037800 & 0.02487000 & -4.39239200 \\
\hline H & 5.32485600 & 0.51096800 & -1.64588800 & F - 4.41208100 & -0.10900200 & -1.72910600 \\
\hline $\mathrm{H}$ & 3.06219300 & 0.93739800 & -2.39061100 & F -0.48911000 & 0.63789400 & 2.41240800 \\
\hline $\mathrm{C}$ & 4.13469800 & 2.73561200 & -0.57106500 & F -0.51677100 & 2.97845200 & 3.69089600 \\
\hline$c$ & 3.00631600 & 3.46491500 & -0.14666100 & F - 2.09617200 & 5.03547700 & 2.80212200 \\
\hline $\mathrm{C}$ & 5.38638500 & 3.37867800 & -0.54070000 & F - -3.64265800 & 4.67765100 & 0.57321900 \\
\hline $\mathrm{C}$ & 3.12460500 & 4.78429300 & 0.29021800 & F -3.62492300 & 2.36034400 & -0.73801700 \\
\hline $\mathrm{H}$ & 2.01935600 & 3.00879500 & -0.15669200 & Si 2.23444300 & -2.42468000 & -0.08996200 \\
\hline $\mathrm{C}$ & 5.50850600 & 4.69963500 & -0.10381700 & H 1.49765200 & -2.80673900 & -1.32014100 \\
\hline $\mathrm{H}$ & 6.27745700 & 2.84507000 & -0.86472000 & H 1.33704300 & -1.85503400 & 0.93901600 \\
\hline $\mathrm{C}$ & 4.37709800 & 5.40349700 & 0.31217300 & H -0.76675800 & -0.44445300 & 0.16932500 \\
\hline H & 2.23883300 & 5.32896300 & 0.60576200 & C 3.10020600 & -3.92368900 & 0.62185300 \\
\hline $\mathrm{H}$ & 6.48431300 & 5.17841800 & -0.09016800 & C 3.69637200 & -4.89279700 & -0.20837900 \\
\hline $\mathrm{H}$ & 4.46937100 & 6.43256900 & 0.65002200 & C 3.17532400 & -4.11118700 & 2.01481900 \\
\hline \multirow[t]{2}{*}{ П } & 3.37015100 & 0.21499500 & 1.88502200 & C 4.35292100 & -5.99895300 & 0.33235600 \\
\hline & & & & H $\quad 3.64317100$ & -4.78899200 & -1.29043800 \\
\hline \multicolumn{2}{|c|}{ Int10 } & & & C $\quad 3.82757500$ & -5.21815800 & 2.56013300 \\
\hline \multicolumn{2}{|c|}{ B -1.89605000} & -0.07372100 & -0.04152700 & H $\quad 2.70993200$ & -3.38984000 & 2.68372100 \\
\hline \multicolumn{2}{|c|}{ C -2.87147600} & -1.24219200 & 0.56761700 & C $\quad 4.41969600$ & -6.16179600 & 1.71819100 \\
\hline \multicolumn{2}{|c|}{ C - 2.45300200} & -2.57508000 & 0.55332500 & $\mathrm{H} \quad 4.80516200$ & -6.73689700 & -0.32524100 \\
\hline \multicolumn{2}{|c|}{ C -3.19697000 } & -3.63045000 & 1.07405200 & H $\quad 3.86949500$ & -5.34766400 & 3.63860400 \\
\hline \multicolumn{2}{|c|}{ C -4.43910300} & -3.37148400 & 1.64120400 & $\mathrm{H} \quad 4.92578000$ & -7.02619100 & 2.14054300 \\
\hline \multicolumn{2}{|c|}{ C -4.91011000} & -2.06459600 & 1.67110600 & $\mathrm{~N} \quad 2.54439000$ & 2.41039100 & 0.93166700 \\
\hline \multicolumn{2}{|c|}{ C -4.13056600} & -1.04225400 & 1.13477100 & C 2.92052200 & 2.41924300 & 2.33454700 \\
\hline \multicolumn{2}{|c|}{ C - 2.03630200} & 0.05162000 & -1.67673100 & C 3.49266300 & 1.26863400 & 2.88039000 \\
\hline \multicolumn{2}{|c|}{ C -0.91024500} & 0.17454300 & -2.48945100 & C $\quad 2.70090600$ & 3.56553000 & 3.10026900 \\
\hline \multicolumn{2}{|c|}{ C -0.94359000} & 0.25332000 & -3.87860100 & C 3.84647400 & 1.26912800 & 4.22910700 \\
\hline \multicolumn{2}{|c|}{ C - 2.16802000} & 0.20116300 & -4.53137400 & H $\quad 3.67530300$ & 0.39968100 & 2.25552900 \\
\hline \multicolumn{2}{|c|}{ C -3.32758700} & 0.07327600 & -3.77461900 & C 3.05534100 & 3.54660800 & 4.44822700 \\
\hline \multicolumn{2}{|c|}{ C -3.23948000 } & 0.00393300 & -2.38644200 & H $\quad 2.23498700$ & 4.44500900 & 2.66877300 \\
\hline \multicolumn{2}{|c|}{ C -2.03773700} & 1.36922000 & 0.74180700 & C 3.62808200 & 2.40408300 & 5.01187100 \\
\hline \multicolumn{2}{|c|}{ C - 2.83010800} & 2.45109000 & 0.34920700 & H 4.29729200 & 0.38191200 & 4.66286200 \\
\hline \multicolumn{2}{|c|}{ C -2.86448500} & 3.67445700 & 1.01561800 & H $\quad 2.87354600$ & 4.42583700 & 5.05851800 \\
\hline \multicolumn{2}{|c|}{ C -2.08607400} & 3.86022000 & 2.15191600 & H 3.90361900 & 2.39905300 & 6.06219700 \\
\hline
\end{tabular}




$\begin{array}{cccc}\text { C } & 3.08964300 & 3.47915600 & 0.06846800 \\ \text { H } & 4.17672700 & 3.47852100 & 0.16113100 \\ \text { H } & 2.79196100 & 3.29200800 & -0.96257200 \\ \text { H } & 2.69436400 & 4.44078600 & 0.40166000 \\ \text { C } & 1.79552400 & 1.48131900 & 0.45492300 \\ \text { O } & 3.31171300 & -1.19234000 & -0.47104700 \\ \text { H } & 1.37034800 & 0.74051700 & 1.12123400 \\ \text { Si } 4.60341600 & -0.98881700 & -1.50355300 \\ \text { H } & 5.86340000 & -0.90088200 & -0.71739700 \\ \text { H } & 4.68285800 & -2.15813000 & -2.42202100 \\ \text { C } & 4.41235100 & 0.60647900 & -2.46636800 \\ \text { C } & 3.26301100 & 0.85760300 & -3.24185000 \\ \text { C } & 5.43111000 & 1.57715900 & -2.45866900 \\ \text { C } & 3.13263000 & 2.03910200 & -3.97382300 \\ \text { H } & 2.45545200 & 0.13266200 & -3.27679100 \\ \text { C } & 5.30866100 & 2.75692400 & -3.19797800 \\ \text { H } & 6.33401600 & 1.41129100 & -1.87413300 \\ \text { C } & 4.15745500 & 2.99024700 & -3.95366500 \\ \text { H } & 2.23487400 & 2.20811300 & -4.56227300 \\ \text { H } & 6.11001000 & 3.49154300 & -3.18503600 \\ \text { H } & 4.06062200 & 3.90716800 & -4.52954200 \\ \text { H } & 1.56430800 & 1.46385100 & -0.60294200\end{array}$

Int11

B -0.05788900

C -1.66888900

C -2.49283200

C -3.87275100

C -4.51247700

C -3.75019800

C -2.37250000

C $\quad 0.67907100$

C 1.64521400

C 2.34060500

C 2.08354600

C 1.13354600

C $\quad 0.46634200$

C $\quad 0.84079100$

C $\quad 0.71074600$

C 1.51458700

C $\quad 2.52772100$

C 2.70931000

C 1.87604900

F - 1.96807600
$-0.01126900$

$-0.10024700$

1.02898900

0.99252200

$-0.23150100$

$-1.39028500$

$-1.30159000$

1.35054200

2.01837000

3.14099600

3.64560300

3.01710400

1.89705300

$-1.28217800$

$-1.86143700$

$-2.89552500$

$-3.39394400$

$-2.84866700$

$-1.81662400$

2.26820400
$-0.90857000$

$-0.58610400$

$-0.54809800$

$-0.36434100$

$-0.22329600$

$-0.27505900$

$-0.46175500$

$-0.34015900$

$-1.09592500$

$-0.64917100$

0.61853500

1.41450800

0.92696900

$-0.36577100$

0.89788400

1.36813900

0.55764100

$-0.70620300$

$-1.13596900$

$-0.70905200$
F -4.59245200

2.13140700

$-0.29424900$

F -5.84500700

F - 4.35181800

F - 1.72754000

F 1.96450000

$-2.59034400$

$-2.49117800$

1.59194100

F 3.25957900

3.74105500

F 2.74503100

4.72631300

F 0.87242900

F - 0.44658500

3.49776600

1.34648300

F 2.13100500

$-1.34098900$

F 3.68944300

$-3.32159700$

F 3.32080300

$-4.39001700$

F 1.32616600

$-3.41661200$

F -0.25344700

H 0.02588200

$-1.43204700$

0.00604100

Int12

$\begin{array}{lccc}\text { N } & 1.62830200 & -0.09402600 & -0.06964900 \\ \text { C } & 0.17831300 & -0.03519700 & -0.03830900 \\ \text { C }-0.53304700 & -1.19363300 & 0.28651700 \\ \text { C }-0.47185100 & 1.16941000 & -0.31995700 \\ \text { C }-1.92531200 & -1.14430200 & 0.31445300 \\ \text { H }-0.00729000 & -2.10550500 & 0.55103600 \\ \text { C }-1.86483200 & 1.19899800 & -0.29078800 \\ \text { H } & 0.08468900 & 2.06124500 & -0.58646300 \\ \text { C }-2.59101400 & 0.04823100 & 0.02502300 \\ \text { H }-2.48586400 & -2.03643300 & 0.57562600 \\ \text { H }-2.38202500 & 2.12568200 & -0.51898500 \\ \text { H }-3.67578600 & 0.08343900 & 0.05269700 \\ \text { C } & 2.36891700 & 0.97497600 & 0.63810000 \\ \text { H } & 2.02180200 & 1.00597500 & 1.67225400 \\ \text { H } & 3.43657800 & 0.76376800 & 0.59406600 \\ \text { H } & 2.16143300 & 1.93053800 & 0.15354600 \\ \text { C } & 2.24561800 & -1.05910900 & -0.65140700 \\ \text { H } & 1.67938200 & -1.81269700 & -1.18819400 \\ \text { H } & 3.32820800 & -1.11406600 & -0.59983300\end{array}$

TS17

$\begin{array}{lrrr}\text { B } & 2.29873200 & -0.35352100 & -0.12438600 \\ \text { C } & 2.90326200 & 1.05444100 & -0.56292500 \\ \text { C } & 2.40400200 & 1.74527700 & -1.67292100 \\ \text { C } & 2.86575800 & 2.98880400 & -2.08162200 \\ \text { C } & 3.90737200 & 3.58699000 & -1.37908900 \\ \text { C } & 4.45471000 & 2.93517000 & -0.28006100\end{array}$

$-0.33126500$

$-0.04736500$

$-0.15628000$

$-0.53403500$

$-2.33878700$

$-1.43155000$

1.07070000

2.64620200

1.76084700

$-2.37589400$

$-1.50149700$

0.99231800

2.59664800

1.74450600

$-2.11754700$

$-0.59983300$ 


\begin{tabular}{|c|c|c|c|c|c|c|c|}
\hline $\mathrm{C}$ & 3.94838700 & 1.69772600 & 0.10830500 & $\mathrm{O}$ & -3.76426600 & 0.47605600 & 0.26351700 \\
\hline $\mathrm{C}$ & 2.11118600 & -1.48266500 & -1.23631900 & $\mathrm{H}$ & -3.87913100 & -1.00302800 & -1.13848800 \\
\hline $\mathrm{C}$ & 1.13162200 & -2.47448100 & -1.12007100 & $\mathrm{O}$ & -5.65053200 & -0.65414100 & -0.24760900 \\
\hline $\mathrm{C}$ & 0.93365400 & -3.47787200 & -2.05932000 & & -6.61994700 & 0.20875400 & 0.90236800 \\
\hline $\mathrm{C}$ & 1.76453600 & -3.53398000 & -3.17456800 & $\mathrm{H}$ & -6.60404000 & 1.64130000 & 0.52916400 \\
\hline $\mathrm{C}$ & 2.76452200 & -2.58148100 & -3.33189000 & $\mathrm{H}$ & -6.04853500 & -0.02844600 & 2.24750000 \\
\hline $\mathrm{C}$ & 2.91841000 & -1.58247000 & -2.37408400 & $\mathrm{C}$ & -8.33453000 & -0.49962700 & 0.76730900 \\
\hline $\mathrm{C}$ & 2.44003100 & -0.81366300 & 1.39647800 & $\mathrm{C}$ & -8.72413200 & -1.60210600 & 1.55126700 \\
\hline $\mathrm{C}$ & 2.95571100 & -2.05653100 & 1.77923600 & $\mathrm{C}$ & -9.27809600 & 0.05673700 & -0.11698600 \\
\hline $\mathrm{C}$ & 3.08569300 & -2.45114600 & 3.10833400 & & 10.00790900 & -2.13787900 & 1.44665200 \\
\hline $\mathrm{C}$ & 2.68633300 & -1.58871000 & 4.12240700 & $\mathrm{H}$ & -8.02136800 & -2.04676400 & 2.25275500 \\
\hline $\mathrm{C}$ & 2.16655700 & -0.33999600 & 3.79415600 & & 10.56254400 & -0.47742000 & -0.22330800 \\
\hline $\mathrm{C}$ & 2.06816800 & 0.02009300 & 2.45686900 & $\mathrm{H}$ & -9.01116500 & 0.91737200 & -0.72685500 \\
\hline $\mathrm{F}$ & 1.39245500 & 1.20976800 & -2.39173200 & & 10.92742400 & -1.57614900 & 0.55798200 \\
\hline $\mathrm{F}$ & 2.32438100 & 3.60958300 & -3.13839500 & & -10.29185100 & -2.98957000 & 2.05912700 \\
\hline $\mathrm{F}$ & 4.37716700 & 4.77878700 & -1.75793600 & & -11.27865900 & -0.03590800 & -0.91133100 \\
\hline $\mathrm{F}$ & 5.46446300 & 3.50009000 & 0.39580600 & & -11.92875500 & -1.99130900 & 0.47782700 \\
\hline $\mathrm{F}$ & 4.53776700 & 1.12433900 & 1.17465400 & & & & \\
\hline $\mathrm{F}$ & 0.29989600 & -2.46948900 & -0.05444200 & Int & & & \\
\hline $\mathrm{F}$ & -0.03558800 & -4.38933100 & -1.89939900 & $\mathrm{~B}$ & 1.88868400 & -0.31454000 & -0.01966700 \\
\hline $\mathrm{F}$ & 1.60054000 & -4.49632500 & -4.08675000 & $\mathrm{C}$ & 2.60797400 & 1.04577200 & -0.53832400 \\
\hline $\mathrm{F}$ & 3.57432000 & -2.63409700 & -4.39808100 & $\mathrm{C}$ & 2.11455200 & 1.70641200 & -1.66527500 \\
\hline $\mathrm{F}$ & 3.91900300 & -0.70625100 & -2.58487100 & $\mathrm{C}$ & 2.62798800 & 2.89610700 & -2.16357500 \\
\hline $\mathrm{F}$ & 1.55666900 & 1.24259500 & 2.19544000 & $\mathrm{C}$ & 3.71978900 & 3.47768000 & -1.52761900 \\
\hline $\mathrm{F}$ & 1.77624000 & 0.49927700 & 4.76289500 & $\mathrm{C}$ & 4.26309800 & 2.85591000 & -0.41100000 \\
\hline $\mathrm{F}$ & 2.79999300 & -1.95445700 & 5.40219600 & $\mathrm{C}$ & 3.70683100 & 1.66603400 & 0.05769400 \\
\hline $\mathrm{F}$ & 3.59474600 & -3.65203800 & 3.41514200 & $\mathrm{C}$ & 1.82506800 & -1.52488200 & -1.10716600 \\
\hline $\mathrm{F}$ & 3.37908200 & -2.93995000 & 0.85519500 & $\mathrm{C}$ & 0.88534700 & -2.54963900 & -0.98639600 \\
\hline & -0.77672700 & 0.52187400 & 0.03759100 & $\mathrm{C}$ & 0.76907100 & -3.61593600 & -1.87069100 \\
\hline $\mathrm{H}$ & -1.33328600 & -0.16167700 & -1.15104100 & $\mathrm{C}$ & 1.64989500 & -3.70328700 & -2.94248600 \\
\hline $\mathrm{H}$ & -1.16658800 & -0.06963200 & 1.33367500 & $\mathrm{C}$ & 2.61911200 & -2.72063400 & -3.10264000 \\
\hline $\mathrm{H}$ & 0.72330900 & 0.15537100 & -0.05709200 & $\mathrm{C}$ & 2.69221600 & -1.66797800 & -2.19254800 \\
\hline $\mathrm{C}$ & -0.92274000 & 2.37860200 & -0.01403500 & $\mathrm{C}$ & 2.28679700 & -0.80095900 & 1.47834400 \\
\hline $\mathrm{C}$ & -0.96524900 & 3.07456900 & -1.23703500 & $\mathrm{C}$ & 3.08339900 & -1.91467900 & 1.75247700 \\
\hline $\mathrm{C}$ & -0.98355300 & 3.12056000 & 1.18036700 & $\mathrm{C}$ & 3.41159000 & -2.32768200 & 3.04216100 \\
\hline $\mathrm{C}$ & -1.06277200 & 4.46613500 & -1.26423300 & $\mathrm{C}$ & 2.94519500 & -1.60549400 & 4.13349400 \\
\hline $\mathrm{H}$ & -0.91800900 & 2.53112800 & -2.17701000 & $\mathrm{C}$ & 2.16119400 & -0.47823500 & 3.91529600 \\
\hline $\mathrm{C}$ & -1.08272600 & 4.51185800 & 1.15356100 & $\mathrm{C}$ & 1.86406100 & -0.10244300 & 2.61025600 \\
\hline $\mathrm{H}$ & -0.95502400 & 2.61135200 & 2.14048100 & $\mathrm{~F}$ & 1.06069900 & 1.17667500 & -2.33620700 \\
\hline $\mathrm{C}$ & -1.12097000 & 5.18604300 & -0.06879300 & $\mathrm{~F}$ & 2.08800700 & 3.48213200 & -3.24527000 \\
\hline $\mathrm{H}$ & -1.09361500 & 4.98754000 & -2.21731200 & $\mathrm{~F}$ & 4.24028600 & 4.62355400 & -1.98773400 \\
\hline $\mathrm{H}$ & -1.13094600 & 5.06866300 & 2.08567500 & $\mathrm{~F}$ & 5.32141000 & 3.40360800 & 0.20793200 \\
\hline $\mathrm{H}$ & -1.19721100 & 6.27013000 & -0.09004600 & $\mathrm{~F}$ & 4.30737500 & 1.12660100 & 1.13943400 \\
\hline $\mathrm{C}$ & -4.35609400 & -0.37063000 & -0.37580300 & $\mathrm{~F}$ & 0.00638100 & -2.53510400 & 0.04916400 \\
\hline
\end{tabular}




\begin{tabular}{|c|c|c|c|c|c|c|}
\hline $\mathrm{F}$ & -0.17284500 & -4.55780800 & -1.69521900 & Si-1.12675400 & 2.68338100 & -1.96383200 \\
\hline $\mathrm{F}$ & 1.56384300 & -4.72115600 & -3.80956400 & H -2.24095500 & 1.86635700 & -1.44900000 \\
\hline $\mathrm{F}$ & 3.48215100 & -2.79737100 & -4.12796500 & H - 1.03568400 & 2.68290700 & -3.43608800 \\
\hline $\mathrm{F}$ & 3.68081800 & -0.77512000 & -2.40874700 & C - 1.07974800 & 4.39433700 & -1.24985000 \\
\hline $\mathrm{F}$ & 1.11565700 & 1.01754900 & 2.46566800 & C - 1.70619300 & 4.70117600 & -0.02515300 \\
\hline $\mathrm{F}$ & 1.70770700 & 0.23590000 & 4.95868800 & C -0.42584700 & 5.42894100 & -1.94954200 \\
\hline $\mathrm{F}$ & 3.24842500 & -1.98836800 & 5.38118700 & C - 1.66967700 & 5.99896700 & 0.48519100 \\
\hline $\mathrm{F}$ & 4.17950300 & -3.41156500 & 3.23801900 & H -2.21899200 & 3.92743800 & 0.53869200 \\
\hline $\mathrm{F}$ & 3.59988200 & -2.65583800 & 0.74872600 & C -0.38966700 & 6.72483900 & -1.43693800 \\
\hline & -1.09848000 & 0.29277600 & 0.16237100 & H $\quad 0.05447900$ & 5.22483300 & -2.90425500 \\
\hline $\mathrm{H}$ & -1.15201600 & -0.33694100 & -1.17057700 & C -1.01166600 & 7.00954500 & -0.21875300 \\
\hline $\mathrm{H}$ & -1.09932900 & -0.45910500 & 1.42449100 & H - 2.15772600 & 6.22115900 & 1.43014800 \\
\hline $\mathrm{H}$ & 0.64122300 & 0.07673800 & 0.09772500 & H $\quad 0.11747000$ & 7.51231500 & -1.98762300 \\
\hline $\mathrm{C}$ & -1.12042100 & 2.15429200 & 0.24446600 & H - 0.98698200 & 8.02040600 & 0.17942100 \\
\hline $\mathrm{C}$ & -1.12233800 & 2.93525700 & -0.92633900 & C $\quad 0.82605100$ & 1.52073400 & -0.40258900 \\
\hline $\mathrm{C}$ & -1.19851500 & 2.80974900 & 1.48723900 & O $\quad 0.34843200$ & 1.80921200 & -1.57553900 \\
\hline $\mathrm{C}$ & -1.19915600 & 4.32687700 & -0.85619500 & H - 0.16390100 & 0.17737500 & 0.05879200 \\
\hline $\mathrm{H}$ & -1.04597100 & 2.46252300 & -1.90127300 & O $\quad 2.02624200$ & 1.06500700 & -0.29854100 \\
\hline $\mathrm{C}$ & -1.27583000 & 4.20102300 & 1.55659700 & Si 3.21363900 & 0.45700400 & -1.42892500 \\
\hline $\mathrm{H}$ & -1.18981700 & 2.23488600 & 2.40910700 & H 2.93629500 & 1.02336300 & -2.76469800 \\
\hline $\mathrm{C}$ & -1.27632600 & 4.96117600 & 0.38520100 & H 3.04800200 & -1.00588500 & -1.37323500 \\
\hline $\mathrm{H}$ & -1.19276200 & 4.91423200 & -1.77059100 & C $\quad 4.83636900$ & 1.04926300 & -0.75465800 \\
\hline $\mathrm{H}$ & -1.33163100 & 4.69098300 & 2.52502000 & C $\quad 5.29981200$ & 0.63577400 & 0.51062900 \\
\hline $\mathrm{H}$ & -1.33290100 & 6.04523300 & 0.43984100 & C $\quad 5.64355400$ & 1.91137600 & -1.51968300 \\
\hline $\mathrm{C}$ & -3.85831900 & -0.27927200 & -0.68442000 & C $\quad 6.53112500$ & 1.07679500 & 0.99231900 \\
\hline $\mathrm{O}$ & -3.14685100 & 0.14955500 & 0.23784800 & H 4.69990400 & -0.02766300 & 1.12991700 \\
\hline $\mathrm{H}$ & -3.43541700 & -0.62444600 & -1.63420900 & C $\quad 6.87881100$ & 2.34769300 & -1.03824500 \\
\hline $\mathrm{O}$ & -5.14927800 & -0.36053700 & -0.61349200 & Н $\quad 5.30876500$ & 2.24500600 & -2.49944400 \\
\hline & -6.08421100 & 0.13151700 & 0.80299200 & C 7.32161400 & 1.93079400 & 0.21789100 \\
\hline $\mathrm{H}$ & -5.78313700 & 1.56510500 & 0.99543400 & H $\quad 6.87630400$ & 0.75290000 & 1.97038600 \\
\hline $\mathrm{H}$ & -5.60475700 & -0.72236000 & 1.90961300 & H $\quad 7.49232100$ & 3.01152000 & -1.64135100 \\
\hline $\mathrm{C}$ & -7.85553200 & -0.18088900 & 0.36935600 & H $\quad 8.28289800$ & 2.27010700 & 0.59484200 \\
\hline $\mathrm{C}$ & -8.46313600 & -1.41577200 & 0.66867000 & H $\quad 0.42460100$ & 1.99091000 & 0.49130000 \\
\hline $\mathrm{C}$ & -8.62722300 & 0.81373200 & -0.26242700 & B -0.77564300 & -0.90672500 & 0.31666600 \\
\hline $\mathrm{C}$ & -9.79660500 & -1.65243600 & 0.33633100 & C - 1.51073900 & -1.25842700 & -1.09371600 \\
\hline $\mathrm{H}$ & -7.89529200 & -2.19825700 & 1.16701100 & C -2.88477400 & -1.41341300 & -1.29635800 \\
\hline $\mathrm{C}$ & -9.96036700 & 0.57634900 & -0.59518000 & C - 3.45609800 & -1.66868100 & -2.54280800 \\
\hline $\mathrm{H}$ & -8.18786300 & 1.78138400 & -0.49421900 & C - -2.64532000 & -1.77861800 & -3.66495700 \\
\hline & 10.54433600 & -0.65696500 & -0.29680500 & C -1.26967700 & -1.63130300 & -3.52173800 \\
\hline & 10.25300700 & -2.60950600 & 0.57325800 & C -0.74727500 & -1.38025800 & -2.25879300 \\
\hline & 10.54400200 & 1.35247600 & -1.08253000 & C -1.75800900 & -0.58694400 & 1.57723800 \\
\hline \multirow{2}{*}{\multicolumn{2}{|c|}{ H - 11.58417300}} & -0.84080500 & -0.55366900 & C -2.07344600 & 0.69091800 & 2.02422900 \\
\hline & & & & C - 2.89160800 & 0.95950500 & 3.11785300 \\
\hline \multicolumn{4}{|c|}{ TS18 } & C -3.43946300 & -0.10125000 & 3.82768700 \\
\hline
\end{tabular}




\begin{tabular}{|c|c|c|c|c|c|}
\hline C -3.16074900 & -1.40385200 & 3.42392200 & H $\quad 1.88681800$ & 0.64041500 & 2.02521200 \\
\hline C - 2.33899100 & -1.61589400 & 2.32217100 & H $\quad 2.04277200$ & 2.21737600 & 0.17030300 \\
\hline C $\quad 0.42818200$ & -1.91264000 & 0.75946100 & C 3.78241900 & 0.06523000 & 0.15172000 \\
\hline C $\quad 0.58772000$ & -3.23292500 & 0.33585900 & C 4.48672300 & 0.46362000 & -1.00018200 \\
\hline C 1.65962500 & -4.03805000 & 0.71561200 & C $\quad 4.38203500$ & -0.88588300 & 0.99750900 \\
\hline C $\quad 2.63822200$ & -3.52564900 & 1.55974300 & C $\quad 5.73793200$ & -0.07494200 & -1.30144500 \\
\hline C $\quad 2.51497800$ & -2.22136300 & 2.02462500 & H $\quad 4.05657400$ & 1.20524300 & -1.67047800 \\
\hline C 1.41893500 & -1.45991900 & 1.63335500 & C $\quad 5.63321100$ & -1.42857700 & 0.69959500 \\
\hline F - 3.75695200 & -1.31995200 & -0.26990800 & H $\quad 3.86753400$ & -1.20717600 & 1.90075700 \\
\hline F - 4.78573900 & -1.80740200 & -2.66709300 & C 6.31202100 & -1.02338700 & -0.45118300 \\
\hline F - 3.17990300 & -2.02269400 & -4.86890600 & Н $\quad 6.26675900$ & 0.24597000 & -2.19532300 \\
\hline F -0.46702300 & -1.73696200 & -4.59326000 & H $\quad 6.07870700$ & -2.16352300 & 1.36518300 \\
\hline F $\quad 0.60230000$ & -1.25874900 & -2.18566600 & H $\quad 7.28778600$ & -1.44236400 & -0.68376900 \\
\hline F - 1.57887700 & 1.78874500 & 1.37990100 & H - 0.89556400 & -0.34082800 & 8300 \\
\hline F - 3.15184000 & 2.22552000 & 3.48591900 & & & \\
\hline F - 4.22921800 & 0.12579600 & 4.88618100 & TS9 & & \\
\hline F - 3.68935300 & -2.43704400 & 4.09803100 & $\mathrm{~N} \quad 1.32881800$ & 2.47381800 & -0.84011800 \\
\hline F - 2.12554800 & -2.90007100 & 1.96393300 & H 2.33679700 & 2.09665600 & -0.25306800 \\
\hline F $\quad 1.34965000$ & -0.20409200 & 2.13627600 & C 1.02101500 & 3.88112800 & -0.55630200 \\
\hline F $\quad 3.46716200$ & -1.69795700 & 2.82124300 & C -0.19549200 & 4.43822600 & -0.95575900 \\
\hline F $\quad 3.68440600$ & -4.27983300 & 1.92419300 & C 1.98082500 & 4.64462100 & 0.10867900 \\
\hline F $\quad 1.75934300$ & -5.30132300 & 0.27119500 & C -0.44579500 & 5.78356400 & -0.68330400 \\
\hline \multirow[t]{2}{*}{ F - 0.31884600} & -3.80370600 & -0.48452200 & H - 0.94212400 & 3.83360 & -1.45800400 \\
\hline & & & C 1.71916200 & 5.98925300 & 0.37505300 \\
\hline Int20 & & & H 2.92106100 & 4.19546300 & 0.41398700 \\
\hline $\mathrm{Si}-2.49306900$ & 1.40763400 & 0.80353300 & C $\quad 0.50821800$ & 6.55929500 & -0.02110400 \\
\hline H - 2.60953900 & 2.67662900 & 0.02878500 & H - 1.39100400 & 6.22269600 & -0.98861300 \\
\hline H - 2.63720700 & 1.66633200 & 2.25581200 & H $\quad 2.46388900$ & 6.58778100 & 0.89133500 \\
\hline C -3.81669900 & 0.23608400 & 0.18525400 & H $\quad 0.30661200$ & 7.60606400 & 0.18741700 \\
\hline C -4.54666700 & 0.52328800 & -0.98291400 & C $\quad 1.33088900$ & 2.10595700 & -2.28236900 \\
\hline C -4.11310600 & -0.95633800 & 0.87438800 & H 2.01010800 & 2.79204300 & -2.78933500 \\
\hline C -5.53442300 & -0.34699500 & -1.44866800 & H 1.68867800 & 1.08181000 & -2.38552300 \\
\hline H -4.34601200 & 1.43880900 & -1.53587900 & H $\quad 0.32862800$ & 2.19083300 & -2.70675900 \\
\hline C -5.09716900 & -1.82941800 & 0.41146200 & C $\quad 0.64421200$ & 1.49986000 & 0.05256500 \\
\hline H -3.57108700 & -1.20738900 & 1.78404200 & O $\quad 0.15976800$ & 0.47961100 & -0.49282900 \\
\hline C -5.80945000 & -1.52475500 & -0.75167700 & O $\quad 2.39728800$ & 1.28127700 & 0.73027700 \\
\hline H -6.08845900 & -0.10637000 & -2.35245500 & H $\quad 0.23468200$ & 1.93481200 & 0.96047300 \\
\hline H -5.31128300 & -2.74471500 & 0.95739900 & B -0.98205500 & -0.54212400 & -0.03803800 \\
\hline H -6.57809100 & -2.20371600 & -1.11211000 & C -0.26658800 & -2.00964700 & 0.06246900 \\
\hline C -0.32548500 & 0.44304300 & -0.55954700 & C $\quad 0.94380700$ & -2.36577300 & -0.53368500 \\
\hline O - 0.94435900 & 0.78305100 & 0.66862600 & C 1.51963500 & -3.62882000 & -0.41497300 \\
\hline H -0.27036500 & 1.33639100 & -1.20269100 & C $\quad 0.86744400$ & -4.61978200 & 0.30516000 \\
\hline O $\quad 0.95259100$ & -0.06507200 & -0.32163600 & C -0.35728700 & -4.32621400 & 0.89446900 \\
\hline Si 2.10577600 & 0.77955300 & 0.56237700 & C -0.89081000 & -3.04972900 & 0.75744200 \\
\hline
\end{tabular}




\begin{tabular}{|c|c|c|c|c|c|}
\hline C - 2.02096600 & -0.41094400 & -1.30244400 & Si-1.10084600 & 0.11399500 & 1.30314500 \\
\hline C -2.48368300 & 0.85007100 & -1.68579900 & H - 1.14470400 & -0.57584100 & 2.61898800 \\
\hline C -3.29662900 & 1.09550800 & -2.78273800 & H -0.69106100 & 1.53234300 & 1.50259900 \\
\hline C -3.71303100 & 0.02284000 & -3.56470400 & C - 2.78949700 & 0.11898500 & 0.49386900 \\
\hline C -3.30068400 & -1.25762000 & -3.22368700 & C -3.46754800 & -1.08001700 & 0.19970800 \\
\hline C -2.47366200 & -1.45183100 & -2.11557400 & C -3.42316200 & 1.33282000 & 0.17217000 \\
\hline C -1.57826000 & -0.07066700 & 1.41838900 & C -4.72914900 & -1.06584300 & -0.39469800 \\
\hline C -2.90517800 & 0.27799000 & 1.69097800 & H -3.00574300 & -2.03719200 & 0.43427900 \\
\hline C -3.36584000 & 0.62546500 & 2.95962000 & C -4.68833000 & 1.35184700 & -0.41925000 \\
\hline C -2.48951600 & 0.63032900 & 4.03722700 & H - 2.92358300 & 2.27601200 & 0.38363100 \\
\hline C - 1.16049800 & 0.28316900 & 3.82696800 & C -5.34201200 & 0.15185000 & -0.70321500 \\
\hline C -0.74801800 & -0.06246100 & 2.54435100 & H -5.23678000 & -2.00158000 & -0.61493300 \\
\hline F $\quad 1.65687600$ & -1.48014100 & -1.27213500 & H -5.16159000 & 2.30053900 & -0.65968600 \\
\hline F $\quad 2.70569600$ & -3.88989100 & -0.99327400 & H -6.32626700 & 0.16368200 & -1.16454400 \\
\hline F $\quad 1.40496500$ & -5.84039100 & 0.42530400 & Si 1.02727900 & -0.65290300 & -0.92396100 \\
\hline F - 1.01447400 & -5.27430400 & 1.58016500 & H 1.02723200 & -2.01348900 & -1.52146300 \\
\hline F - 2.10152600 & -2.84809500 & 1.32334400 & H $\quad 0.52048300$ & 0.33935200 & -1.91254700 \\
\hline F - 2.13915500 & 1.93835000 & -0.94757800 & C $\quad 2.77623400$ & -0.17862800 & -0.45794000 \\
\hline F -3.68308700 & 2.34540700 & -3.08712200 & C 3.69203700 & -1.13851700 & 0.01332400 \\
\hline F -4.50022600 & 0.22444800 & -4.62810600 & C 3.21525600 & 1.15388700 & -0.57108000 \\
\hline F -3.69523000 & -2.30375400 & -3.96502700 & C 4.99348100 & -0.78000700 & 0.36640100 \\
\hline F - 2.13005200 & -2.73364700 & -1.87819400 & H $\quad 3.38901500$ & -2.17992800 & 0.10359300 \\
\hline F $\quad 0.55487600$ & -0.40549800 & 2.41373000 & C 4.51619200 & 1.51732300 & -0.21923300 \\
\hline F -0.29562700 & 0.27651300 & 4.85194800 & H $\quad 2.53518000$ & 13300 & -0.94 \\
\hline F - 2.91873900 & 0.96586700 & 5.25904800 & C $\quad 5.40639300$ & 0.54956100 & 0.25087600 \\
\hline F - 4.65358500 & 0.95128000 & 3.14774200 & H $\quad 5.68611700$ & -1.53581500 & 0.72800900 \\
\hline F -3.84330600 & 0.28092200 & 0.72316600 & H $\quad 4.83590500$ & 2.55196200 & -0.31444700 \\
\hline Si 3.47130400 & 0.11693500 & 1.28053700 & H $\quad 6.42085300$ & 0.82958900 & 0.52338000 \\
\hline Н 3.91934400 & 0.50393400 & 2.64824200 & & & \\
\hline H 2.83537400 & -1.21961500 & 1.33062400 & $3 a$ & & \\
\hline C 4.97310800 & 0.02579100 & 0.15975000 & $\mathrm{~N} \quad 1.57253700$ & 0.00224400 & -0.15827400 \\
\hline С $\quad 6.03720800$ & 0.93745400 & 0.29763800 & C $\quad 0.18391800$ & -0.00045000 & -0.07161100 \\
\hline C $\quad 5.06967300$ & -0.95553900 & -0.84553900 & C -0.55291400 & 1.20774800 & -0.04322700 \\
\hline C 7.14973900 & 0.88231800 & -0.54311400 & C -0.55130400 & -1.20878100 & -0.02197200 \\
\hline H $\quad 6.00282400$ & 1.69735400 & 1.07665700 & C - 1.94506000 & 1.19771500 & 0.00589200 \\
\hline C 6.18278700 & -1.01574000 & -1.68639500 & H -0.03947500 & 2.16187000 & -0.06083900 \\
\hline H $\quad 4.27204200$ & -1.68316300 & -0.97332700 & C -1.94362300 & -1.19935000 & 0.02757000 \\
\hline C 7.22273800 & -0.09554500 & -1.53809800 & H - 0.03749700 & -2.16276800 & -0.01730000 \\
\hline H $\quad 7.96120100$ & 1.59522600 & -0.41901800 & C -2.65955400 & -0.00112400 & 0.03926100 \\
\hline H $\quad 6.24033500$ & -1.78326200 & -2.45424000 & H - 2.47468400 & 2.14767800 & 0.02266800 \\
\hline \multirow[t]{2}{*}{ H 8.09013600} & -0.14344100 & -2.19176500 & H - 2.47192700 & -2.14950600 & 0.06404900 \\
\hline & & & H -3.74506900 & -0.00143900 & 0.08038800 \\
\hline $\mathrm{SiOSi}$ & & & C $\quad 2.29936200$ & -1.24065300 & 0.04244900 \\
\hline O $\quad 0.01819100$ & -0.70286500 & 0.39256700 & H 1.97653300 & -2.00238700 & -0.67573400 \\
\hline
\end{tabular}




\begin{tabular}{|c|c|c|c|c|c|c|c|}
\hline $\mathrm{H}$ & 3.36295800 & -1.06187300 & -0.13044500 & $\mathrm{C}$ & -4.77181600 & -0.92439100 & 2.01729200 \\
\hline $\mathrm{H}$ & 2.17972200 & -1.65455600 & 1.05763200 & $\mathrm{C}$ & -3.76544100 & -0.12649300 & 2.55188900 \\
\hline $\mathrm{C}$ & 2.29326400 & 1.24251500 & 0.08153900 & $\mathrm{C}$ & -2.55536000 & -0.02292400 & 1.87760600 \\
\hline $\mathrm{H}$ & 2.01225000 & 2.00943600 & -0.64940500 & $\mathrm{~F}$ & -2.02291000 & 0.10113700 & -2.56913900 \\
\hline $\mathrm{H}$ & 2.12112200 & 1.65270800 & 1.09011200 & $\mathrm{~F}$ & -1.56888300 & -1.10723100 & -4.91592600 \\
\hline \multirow[t]{2}{*}{$\mathrm{H}$} & 3.36376900 & 1.05941300 & -0.03262400 & $\mathrm{~F}$ & 0.15070900 & -3.23322600 & -5.06648100 \\
\hline & & & & $\mathrm{F}$ & 1.40139300 & -4.11569700 & -2.79604600 \\
\hline \multicolumn{2}{|c|}{ TS4 } & & & $\mathrm{F}$ & 0.97191700 & -2.94823100 & -0.45972900 \\
\hline $\mathrm{C}$ & 0.22828300 & -1.78615600 & 1.89789000 & $\mathrm{~F}$ & 1.91770200 & 0.66077200 & -0.39680100 \\
\hline $\mathrm{O}$ & 0.28428600 & -0.84762900 & 1.02013800 & $\mathrm{~F}$ & 2.57298200 & 3.19642800 & -0.80519900 \\
\hline $\mathrm{O}$ & 1.20231900 & -2.08020900 & 2.61125900 & $\mathrm{~F}$ & 0.63510500 & 5.12263400 & -1.06237600 \\
\hline $\mathrm{H}$ & -0.71711200 & -2.33462600 & 2.01199000 & $\mathrm{~F}$ & -1.99989600 & 4.40866100 & -0.86212800 \\
\hline $\mathrm{H}$ & 2.66916900 & -1.84628800 & 1.48644000 & $\mathrm{~F}$ & -2.69497200 & 1.88739000 & -0.41166400 \\
\hline $\mathrm{Si}$ & 3.22371600 & -0.48899000 & 2.67691500 & $\mathrm{~F}$ & -1.61694900 & 0.76658200 & 2.45121400 \\
\hline $\mathrm{H}$ & 2.08735300 & 0.41095900 & 2.48425000 & $\mathrm{~F}$ & -3.97143200 & 0.52930800 & 3.70538500 \\
\hline $\mathrm{H}$ & 3.34135400 & -1.57668300 & 1.19781800 & $\mathrm{~F}$ & -5.94750700 & -1.04397300 & 2.64709300 \\
\hline $\mathrm{H}$ & 3.35252000 & -1.39813400 & 3.82098900 & $\mathrm{~F}$ & -5.49521400 & -2.38234700 & 0.30547100 \\
\hline $\mathrm{C}$ & 4.84963100 & 0.21220700 & 2.17164500 & $\mathrm{~F}$ & -3.17322400 & -2.17830700 & -0.95308700 \\
\hline $\mathrm{C}$ & 4.94466400 & 1.25914800 & 1.22983300 & & & & \\
\hline $\mathrm{C}$ & 6.02913900 & -0.27641800 & 2.77310300 & TS5 & & & \\
\hline $\mathrm{C}$ & 6.18619100 & 1.80126200 & 0.90672700 & $\mathrm{C}$ & 3.53298400 & 0.50255800 & 0.45869500 \\
\hline $\mathrm{H}$ & 4.05511600 & 1.64528500 & 0.74064000 & $\mathrm{O}$ & 2.77720600 & 1.37102700 & 0.86120100 \\
\hline $\mathrm{C}$ & 7.26780900 & 0.27137300 & 2.44681900 & $\mathrm{O}$ & 3.15342000 & -0.67830600 & -0.01391800 \\
\hline $\mathrm{H}$ & 5.97950200 & -1.08150600 & 3.50293400 & $\mathrm{H}$ & 4.62984900 & 0.63628000 & 0.45602100 \\
\hline $\mathrm{C}$ & 7.34490400 & 1.30889100 & 1.51419400 & $\mathrm{H}$ & 2.09945300 & -1.08900700 & 0.72045100 \\
\hline $\mathrm{H}$ & 6.25101000 & 2.60699900 & 0.18123700 & Si 0 & 0.99292200 & -0.63331600 & -0.65614600 \\
\hline $\mathrm{H}$ & 8.16995200 & -0.10793000 & 2.91806400 & $\mathrm{H}$ & 1.44110100 & 0.54592100 & -1.42463300 \\
\hline $\mathrm{H}$ & 8.31115000 & 1.73618400 & 1.26024100 & $\mathrm{H}$ & 1.21732000 & -1.26283500 & 0.91111400 \\
\hline $\mathrm{B}$ & -0.78721300 & -0.45796600 & -0.01593200 & $\mathrm{H}$ & 1.19302000 & -1.91738800 & -1.35742400 \\
\hline $\mathrm{C}$ & -0.52643800 & -1.31996500 & -1.39396400 & $\mathrm{C}-\mathrm{C}$ & 0.79412900 & -0.27433300 & -0.22285300 \\
\hline $\mathrm{C}$ & -1.14535300 & -0.92647700 & -2.58444700 & $\mathrm{C}-1$ & 1.37454500 & 0.92485200 & -0.67336000 \\
\hline $\mathrm{C}$ & -0.93859300 & -1.54123500 & -3.81313500 & $\mathrm{C}-1$ & 1.59929500 & -1.17793100 & 0.49636100 \\
\hline $\mathrm{C}$ & -0.06803500 & -2.62332200 & -3.89353200 & $\mathrm{C}-2$ & 2.71942900 & 1.20822500 & -0.42482900 \\
\hline $\mathrm{C}$ & 0.56356100 & -3.06639600 & -2.74015600 & $\mathrm{H}-\mathrm{C}$ & 0.77153000 & 1.64747900 & -1.21870800 \\
\hline $\mathrm{C}$ & 0.32159700 & -2.41883600 & -1.52894500 & $\mathrm{C}-2$ & 2.94203100 & -0.89920700 & 0.74339600 \\
\hline $\mathrm{C}$ & -0.43278800 & 1.12771800 & -0.32447900 & $\mathrm{H}-1$ & 1.17469200 & -2.10781900 & 0.87103100 \\
\hline $\mathrm{C}$ & 0.89137300 & 1.54923600 & -0.47305600 & $\mathrm{C}-3$ & 3.50332400 & 0.29584200 & 0.28205000 \\
\hline $\mathrm{C}$ & 1.27011400 & 2.86721700 & -0.70641300 & $\mathrm{H}-3$ & 3.15118500 & 2.14100300 & -0.77791300 \\
\hline $\mathrm{C}$ & 0.29434900 & 3.84703000 & -0.83545200 & $\mathrm{H}-3$ & 3.55146900 & -1.60869800 & 1.29700800 \\
\hline $\mathrm{C}$ & -1.04082300 & 3.47890200 & -0.72988200 & $\mathrm{H}-4$ & 4.54926600 & 0.51586400 & 0.47961200 \\
\hline $\mathrm{C}$ & -1.37370200 & 2.14768700 & -0.48551400 & & & & \\
\hline $\mathrm{C}$ & -2.26480500 & -0.67435800 & 0.67331100 & $12 \mathrm{a}$ & & & \\
\hline $\mathrm{C}$ & -3.30501000 & -1.46817700 & 0.18705000 & $\mathrm{C}-1$ & 1.08050000 & 0.08300600 & -1.95834300 \\
\hline $\mathrm{C}$ & -4.53768400 & -1.59968700 & 0.82765400 & $\mathrm{H}-1$ & 1.38516300 & 0.00914200 & -3.01424300 \\
\hline
\end{tabular}




\begin{tabular}{|c|c|c|c|c|c|}
\hline O $\quad 0.20102200$ & 0.01993200 & -1.81403800 & H -4.65386900 & 0.90872700 & -2.22182300 \\
\hline В 0.90770300 & 0.07790200 & -0.44930800 & C -7.72407800 & -0.57599800 & -2.24026200 \\
\hline C $\quad 0.49453400$ & 1.54277100 & 0.18815400 & H -8.50036600 & -1.83506700 & -0.66849900 \\
\hline C $\quad 0.94941700$ & 2.72358700 & -0.40689800 & H -6.68791800 & 0.69908900 & -3.63980400 \\
\hline C $\quad 0.57091500$ & 4.00057200 & -0.00269000 & H -8.60928800 & -0.67258600 & -2.86185800 \\
\hline C -0.33414400 & 4.14606200 & 1.04188700 & C -4.30170100 & 0.97999100 & 1.12905700 \\
\hline C -0.83267700 & 3.00599700 & 1.65664100 & H -5.14807600 & 0.85933800 & 1.80592300 \\
\hline C -0.41573000 & 1.75032400 & 1.22331200 & H -3.36099700 & 0.99642100 & 1.67862200 \\
\hline C $\quad 2.51236800$ & -0.09245100 & -0.79496800 & H -4.41477800 & 1.89107500 & 0.54133600 \\
\hline C 2.93587300 & -1.07711900 & -1.69367100 & H -3.41206600 & -0.08408100 & -0.42557400 \\
\hline C $\quad 4.27016100$ & -1.31817500 & -2.00937500 & & & \\
\hline C $\quad 5.26398100$ & -0.56996900 & -1.39086200 & Int13 & & \\
\hline C 4.89995000 & 0.40046900 & -0.46688300 & C $\quad 0.39276000$ & 0.33143500 & -2.66354500 \\
\hline C $\quad 3.55206900$ & 0.61148100 & -0.18386400 & O $\quad 0.23334800$ & -0.31192600 & -1.56346900 \\
\hline C $\quad 0.53787500$ & -1.22927100 & 0.48921900 & O -0.30381000 & 0.11813100 & -3.67532200 \\
\hline C 1.03956400 & -1.29738000 & 1.79225600 & H 1.17330100 & 1.09896400 & -2.71656800 \\
\hline C $\quad 0.87664000$ & -2.38617800 & 2.64036400 & H - 1.36138900 & -0.77989600 & -3.44610200 \\
\hline C $\quad 0.18681900$ & -3.50426200 & 2.18385000 & B 1.02533900 & -0.01708200 & -0.26499700 \\
\hline C -0.31972100 & -3.49878000 & 0.89191400 & C $\quad 2.57008200$ & -0.48824800 & -0.59763400 \\
\hline C -0.12917600 & -2.38307700 & 0.07559700 & C 3.04431200 & -1.78096400 & -0.35471700 \\
\hline F $\quad 1.80909500$ & 2.67038200 & -1.44746000 & C 4.32395300 & -2.21610700 & -0.69408200 \\
\hline F $\quad 1.05975300$ & 5.08965300 & -0.61954300 & C 5.19913700 & -1.34621000 & -1.33044300 \\
\hline F - 0.72691900 & 5.36474500 & 1.44087300 & C $\quad 4.77224500$ & -0.05656200 & -1.62235800 \\
\hline F - 1.74347400 & 3.11580900 & 2.64416100 & C 3.48662400 & 0.33246500 & -1.26114100 \\
\hline F - 1.00233600 & 0.70783800 & 1.86428500 & C $\quad 0.30779900$ & -0.88368700 & 0.93924000 \\
\hline F $\quad 2.03581600$ & -1.88491500 & -2.29659400 & C -0.93088500 & -1.52317900 & 0.88406000 \\
\hline F $\quad 4.60511100$ & -2.27614400 & -2.89158600 & C -1.50930700 & -2.18179600 & 1.96838600 \\
\hline F $\quad 6.55649000$ & -0.78731800 & -1.67615400 & C -0.85729500 & -2.21242300 & 3.19184300 \\
\hline F $\quad 5.84991000$ & 1.12650600 & 0.14748600 & C $\quad 0.37867400$ & -1.58473800 & 3.30438100 \\
\hline F $\quad 3.29798000$ & 1.56476000 & 0.73883000 & C $\quad 0.91914500$ & -0.94293500 & 2.19615300 \\
\hline F -0.65166500 & -2.49280300 & -1.16884600 & C $\quad 0.81618500$ & 1.57060000 & 0.14736300 \\
\hline F - 0.98939200 & -4.57205500 & 0.43241700 & C $\quad 1.76108800$ & 2.35824000 & 0.81277500 \\
\hline F $\quad 0.01181300$ & -4.56968000 & 2.98046200 & C 1.52129300 & 3.66571200 & 1.23006900 \\
\hline F $\quad 1.37770000$ & -2.37151100 & 3.88702200 & C $\quad 0.28015600$ & 4.24632800 & 1.00101800 \\
\hline F $\quad 1.72868000$ & -0.24739500 & 2.29353000 & C -0.70233900 & 3.50317100 & 0.35918600 \\
\hline O - 1.91590200 & 0.22699200 & -1.06196000 & C -0.41979300 & 2.19924900 & -0.03590000 \\
\hline N -4.26620100 & -0.18747000 & 0.18059800 & F $\quad 2.25767300$ & -2.71153200 & 0.22846800 \\
\hline H -4.11912500 & -1.04577800 & 0.72351000 & F $\quad 4.71102000$ & -3.47248700 & -0.42146300 \\
\hline C -5.46688800 & -0.33708500 & -0.66047700 & F $\quad 6.43063200$ & -1.74743500 & -1.67049400 \\
\hline C -6.53000600 & -1.11175900 & -0.20459600 & F $\quad 5.59501600$ & 0.79494300 & -2.25492600 \\
\hline C -5.50358600 & 0.32053600 & -1.88711800 & F $\quad 3.13990100$ & 1.59465100 & -1.61955300 \\
\hline C -7.66538900 & -1.22936700 & -1.00731900 & F - 1.67101800 & -1.55393300 & -0.25010700 \\
\hline H -6.47368300 & -1.62042800 & 0.75438000 & F - 2.71190300 & -2.78020900 & 1.83424300 \\
\hline C -6.64620500 & 0.19618800 & -2.67852300 & F - 1.40471600 & -2.83660500 & 4.24352800 \\
\hline
\end{tabular}




\begin{tabular}{|c|c|c|c|c|c|}
\hline F $\quad 1.03284900$ & -1.60086100 & 4.47610800 & Н 1.37120200 & 2.59741600 & 0.52706500 \\
\hline F $\quad 2.12153500$ & -0.35442200 & 2.37753700 & H $\quad 2.80421800$ & 1.78483400 & -0.12037300 \\
\hline F - 1.44892400 & 1.53329300 & -0.62362600 & O $\quad 2.50629300$ & -1.22690700 & -0.68409100 \\
\hline F - 1.91381000 & 4.03986100 & 0.13660800 & Н 3.12654300 & -1.93796700 & -0.44685700 \\
\hline F $\quad 0.03304600$ & 5.50255500 & 1.39479800 & H 3.05922200 & -0.08316700 & 0.97067800 \\
\hline F $\quad 2.47956200$ & 4.36936300 & 1.85277200 & & & \\
\hline F 2.99104900 & 1.88031700 & 1.08670700 & \multicolumn{3}{|l|}{ Int15 } \\
\hline N -4.10215500 & -1.31611800 & -1.96883200 & C $\quad 0.65992300$ & 0.09473800 & 1.99118700 \\
\hline C -5.03748300 & -0.53205100 & -1.19393200 & O $\quad 0.26764600$ & -0.30434100 & 0.84262400 \\
\hline C -5.84674400 & 0.40334200 & -1.83957400 & O $\quad 1.83799200$ & -0.08616000 & 2.37791700 \\
\hline C -5.11893900 & -0.73516400 & 0.18568000 & H - 0.04533700 & 0.59766500 & 2.66129200 \\
\hline C -6.75029000 & 1.15541100 & -1.08650400 & B -1.09855100 & -0.00116500 & 0.16090300 \\
\hline H -5.77530300 & 0.53072300 & -2.91584700 & C - 2.24529900 & -0.10625200 & 1.32812100 \\
\hline C -6.03345700 & 0.01494400 & 0.92443300 & C -2.40261500 & -1.31403400 & 2.01864800 \\
\hline H - 4.46049200 & -1.44806100 & 0.67139600 & C -3.32937900 & -1.51875000 & 3.03312300 \\
\hline C -6.84698400 & 0.95879600 & 0.29181800 & C -4.16186400 & -0.47017400 & 3.41120100 \\
\hline H -7.38130500 & 1.88806000 & -1.58100400 & C -4.04300300 & 0.75397000 & 2.76785600 \\
\hline H -6.10045900 & -0.13127500 & 1.99851100 & C -3.09609100 & 0.91492600 & 1.75583200 \\
\hline H -7.55421400 & 1.54207800 & 0.87441300 & C -1.19849600 & -1.17087900 & -0.99829800 \\
\hline C -4.25654100 & -2.78201500 & -2.03377400 & C -0.09416900 & -1.51402300 & -1.78005500 \\
\hline H -5.29443000 & -3.02754900 & -1.81260300 & C -0.10320000 & -2.47321900 & -2.78600300 \\
\hline H -3.99749100 & -3.13239200 & -3.03410500 & C - 1.28909900 & -3.13848000 & -3.07232900 \\
\hline H -3.59472700 & -3.25257100 & -1.30091900 & C -2.42889500 & -2.82485800 & -2.34274300 \\
\hline C -3.06254100 & -0.73353100 & -2.51623800 & C -2.36581900 & -1.85932500 & -1.33951800 \\
\hline O - 2.21799800 & -1.39177300 & -3.21526200 & C -0.99627700 & 1.44781400 & -0.60929700 \\
\hline \multirow[t]{2}{*}{ H - 2.93973400} & 0.33670600 & -2.35271200 & C -2.04413200 & 1.84132600 & -1.44808100 \\
\hline & & & C -2.04003700 & 3.00195800 & -2.21124300 \\
\hline Int14 & & & C -0.93407500 & 3.84537800 & -2.16018600 \\
\hline $\mathrm{N} \quad 1.21542500$ & 0.49260400 & 0.27197100 & C $\quad 0.13477700$ & 3.50590000 & -1.34287100 \\
\hline C -0.14309500 & 0.19480600 & 0.12447900 & C $\quad 0.08062000$ & 2.33179500 & -0.59334400 \\
\hline C - 1.09379300 & 1.22828600 & -0.01623000 & F - 1.61786500 & -2.36842700 & 1.70148900 \\
\hline C -0.61556300 & -1.13615500 & 0.12698000 & F - 3.42539300 & -2.70610000 & 3.65043600 \\
\hline C -2.45422600 & 0.93859300 & -0.11508700 & F -5.05908000 & -0.63815500 & 4.38967500 \\
\hline H -0.77463800 & 2.26430200 & -0.03605900 & F -4.82934200 & 1.77842300 & 3.13217000 \\
\hline C -1.97835400 & -1.40816000 & 0.03624800 & F -3.04170800 & 2.14805100 & 1.21001400 \\
\hline H $\quad 0.08698400$ & -1.96069700 & 0.15313200 & F 1.11066000 & -0.88409400 & -1.59836800 \\
\hline C -2.91425800 & -0.37801400 & -0.08132300 & F $\quad 1.01081300$ & -2.74829200 & -3.48246300 \\
\hline H -3.15986500 & 1.75992300 & -0.21684500 & F - 1.33169500 & -4.06354800 & -4.03837400 \\
\hline H - 2.30739600 & -2.44480700 & 0.03938200 & F - 3.58321300 & -3.45156700 & -2.61119200 \\
\hline H -3.97526900 & -0.59808500 & -0.15841700 & F - 3.52421100 & -1.61487800 & -0.69652500 \\
\hline C 2.16413800 & -0.54031100 & 0.52850000 & F $\quad 1.17924300$ & 2.09734300 & 0.18081700 \\
\hline H 1.74659500 & -1.24209100 & 1.26262900 & F $\quad 1.21090800$ & 4.30669500 & -1.27539800 \\
\hline C 1.71270500 & 1.79747200 & -0.14354400 & F - 0.90341200 & 4.96891500 & -2.88740400 \\
\hline H 1.39876300 & 2.04827300 & -1.16553800 & F - 3.08374800 & 3.31623200 & -2.99216800 \\
\hline
\end{tabular}




\begin{tabular}{|c|c|c|c|c|c|c|}
\hline \multicolumn{2}{|c|}{ F - 3.14850300} & \multirow{2}{*}{$\begin{array}{l}1.06831200 \\
0.63313400\end{array}$} & \multirow{2}{*}{$\begin{array}{r}-1.52974500 \\
0.64078300\end{array}$} & \multirow{2}{*}{$\begin{array}{ll}\mathrm{C} & 1.90151100 \\
\mathrm{C} & 0.73885500\end{array}$} & \multirow{2}{*}{$\begin{array}{l}-3.07396300 \\
-3.83865100\end{array}$} & \multirow{2}{*}{$\begin{array}{l}-2.12115000 \\
-2.09808300\end{array}$} \\
\hline $\mathrm{N}$ & 4.90622800 & & & & & \\
\hline $\mathrm{C}$ & 6.13311200 & -0.05882300 & 0.77874500 & C -0.32829300 & -3.41349200 & -1.32035100 \\
\hline $\mathrm{C}$ & 7.22333000 & 0.55372400 & 1.42151800 & C -0.21348100 & -2.23647000 & -0.58230700 \\
\hline $\mathrm{C}$ & 6.29570800 & -1.36376700 & 0.27700000 & F $\quad 1.86138400$ & 2.41842900 & 1.59778800 \\
\hline $\mathrm{C}$ & 8.44306100 & -0.11364500 & 1.53298600 & F $\quad 3.70255100$ & 2.68014800 & 3.52134200 \\
\hline $\mathrm{H}$ & 7.13256000 & 1.55369400 & 1.82911200 & F $\quad 5.15261000$ & 0.51129200 & 4.35226500 \\
\hline $\mathrm{C}$ & 7.52345300 & -2.01388200 & 0.38686200 & F $\quad 4.69486700$ & -1.93265700 & 3.21086700 \\
\hline $\mathrm{H}$ & 5.46109500 & -1.89146800 & -0.17044800 & F $\quad 2.86476400$ & -2.22903100 & 1.31678900 \\
\hline $\mathrm{C}$ & 8.60787000 & -1.39683100 & 1.01230200 & F - 1.08353000 & 1.00910600 & -1.56138600 \\
\hline $\mathrm{H}$ & 9.27099600 & 0.38404800 & 2.03117400 & F - 0.89458800 & 2.78483900 & -3.52287500 \\
\hline $\mathrm{H}$ & 7.62178400 & -3.02158900 & -0.00841600 & F $\quad 1.52029700$ & 3.91545900 & -4.16754200 \\
\hline $\mathrm{H}$ & 9.55976600 & -1.91200600 & 1.10220400 & F $\quad 3.75157700$ & 3.20874000 & -2.75021800 \\
\hline $\mathrm{C}$ & 3.92054300 & 0.17413700 & -0.21414500 & F $\quad 3.60562600$ & 1.45597600 & -0.76629100 \\
\hline $\mathrm{H}$ & 4.30948600 & -0.32397700 & -1.10078000 & F - 1.31885300 & -1.91742300 & 0.15339800 \\
\hline $\mathrm{C}$ & 4.65334300 & 1.83018800 & 1.44576900 & F - 1.46287700 & -4.13439500 & -1.28034800 \\
\hline $\mathrm{H}$ & 4.79046300 & 1.62328100 & 2.51265900 & F $\quad 0.65241200$ & -4.96730800 & -2.81486500 \\
\hline $\mathrm{H}$ & 5.31961600 & 2.65116600 & 1.15635700 & F $\quad 2.94535300$ & -3.47020900 & -2.86526900 \\
\hline $\mathrm{H}$ & 3.62540800 & 2.15892400 & 1.28789700 & F 3.12061100 & -1.21402800 & -1.43117200 \\
\hline $\mathrm{O}$ & 3.06912200 & -0.95294700 & 0.45162200 & N -4.93849600 & -0.57172900 & 0.59852700 \\
\hline $\mathrm{H}$ & 2.34394800 & -1.17456200 & -0.17577200 & C -6.22992700 & 0.06407100 & 0.75226900 \\
\hline $\mathrm{H}$ & 3.20394700 & 0.95094700 & -0.46963900 & C -7.38360500 & -0.72162700 & 0.81500300 \\
\hline \multirow[t]{2}{*}{$\mathrm{H}$} & 2.53294500 & -0.56870700 & 1.40084400 & C -6.29792900 & 1.45742900 & 0.83304200 \\
\hline & & & & C -8.62259900 & -0.09662800 & 0.94996100 \\
\hline \multicolumn{2}{|c|}{ Int16 } & & & H -7.32304300 & -1.80253400 & 0.73909900 \\
\hline \multicolumn{2}{|c|}{ C - 0.61335100} & -0.07794600 & 2.03222000 & C -7.54551500 & 2.06823500 & 0.96641000 \\
\hline \multicolumn{2}{|c|}{ O -0.21729000} & 0.42299500 & 0.89709700 & H -5.38578200 & 2.04502400 & 0.80946300 \\
\hline \multicolumn{2}{|c|}{ O -1.69533900 } & 0.20692400 & 2.54356500 & C -8.70629800 & 1.29583100 & 1.02524700 \\
\hline $\mathrm{H}$ & 0.07380300 & -0.77652000 & 2.53318100 & H -9.52395400 & -0.70118000 & 0.98965400 \\
\hline B & 1.08399800 & 0.03035900 & 0.19079600 & H - 7.60412600 & 3.15063100 & 1.03333300 \\
\hline $\mathrm{C}$ & 2.27754600 & 0.09217100 & 1.32506900 & H -9.67444900 & 1.77632200 & 1.13261700 \\
\hline $\mathrm{C}$ & 2.54508000 & 1.31040800 & 1.96055100 & C -4.08317300 & -0.12847300 & -0.27649900 \\
\hline $\mathrm{C}$ & 3.49459000 & 1.47737400 & 2.96142900 & H -4.43011600 & 0.55465900 & -1.03972700 \\
\hline $\mathrm{C}$ & 4.23438000 & 0.37855900 & 3.38573300 & C -4.55671600 & -1.60188400 & 1.58408700 \\
\hline $\mathrm{C}$ & 4.00225700 & -0.85759600 & 2.79964500 & H - 4.49300800 & -1.14477100 & 2.57451700 \\
\hline $\mathrm{C}$ & 3.03749200 & -0.97790000 & 1.79901100 & H -5.30979700 & -2.39184100 & 1.59465700 \\
\hline $\mathrm{C}$ & 1.24787800 & 1.15554500 & -1.01147400 & H -3.59176300 & -2.02514900 & 1.30758200 \\
\hline $\mathrm{C}$ & 0.15455600 & 1.54254500 & -1.78877600 & O -2.99003900 & 1.56394500 & 0.56257500 \\
\hline $\mathrm{C}$ & 0.21147000 & 2.46089000 & -2.83150000 & H - 2.24401000 & 1.64857800 & -0.05965300 \\
\hline $\mathrm{C}$ & 1.43308700 & 3.03278200 & -3.16344400 & H -3.14526000 & -0.65198000 & -0.40076400 \\
\hline $\mathrm{C}$ & 2.56176700 & 2.67128300 & -2.43936900 & H - 2.55900800 & 1.16677600 & 1.36827200 \\
\hline $\mathrm{C}$ & 2.45189900 & 1.75021100 & -1.39904800 & & & \\
\hline $\mathrm{C}$ & 0.92095500 & -1.42927300 & -0.56804700 & Int17 & & \\
\hline $\mathrm{C}$ & 1.96281100 & -1.90660600 & -1.37031800 & C 1.20390100 & -1.65320800 & 0.22942400 \\
\hline
\end{tabular}




\begin{tabular}{|c|c|c|c|c|c|c|}
\hline O $\quad 0.58599000$ & -0.69112400 & -0.38105200 & $\mathrm{H}$ & 4.74796200 & -2.44371400 & 0.18483800 \\
\hline O $\quad 2.34619400$ & -2.01079200 & -0.06529500 & $\mathrm{C}$ & 8.10677300 & -1.90858600 & -0.08690800 \\
\hline H $\quad 0.65912500$ & -2.16793900 & 1.03608600 & $\mathrm{H}$ & 9.01282800 & -0.06250100 & -0.74390800 \\
\hline B -0.80018300 & -0.15713600 & 0.00875200 & $\mathrm{H}$ & 6.92486800 & -3.60621200 & 0.52685400 \\
\hline C -1.77474800 & -1.47052000 & 0.20964300 & $\mathrm{H}$ & 9.05660400 & -2.41156600 & 0.06996800 \\
\hline C - 1.94570900 & -2.35359800 & -0.86295600 & $\mathrm{C}$ & 3.48457600 & -0.50366900 & -1.41924500 \\
\hline C -2.71410400 & -3.51001600 & -0.80878000 & $\mathrm{H}$ & 3.71830600 & -1.38740800 & -1.99720900 \\
\hline C -3.36118200 & -3.83970600 & 0.37753600 & $\mathrm{C}$ & 4.16868500 & 1.29693800 & 0.04096100 \\
\hline C -3.21908900 & -3.00565200 & 1.47732000 & $\mathrm{H}$ & 4.13318300 & 1.07325800 & 1.10941000 \\
\hline C - 2.43544200 & -1.85548400 & 1.37709500 & $\mathrm{H}$ & 4.98275000 & 1.99598200 & -0.15826400 \\
\hline C -1.22470800 & 0.79975200 & -1.27083100 & $\mathrm{H}$ & 3.22200600 & 1.72623200 & -0.28244600 \\
\hline C -0.30355700 & 1.69103200 & -1.82647000 & $\mathrm{H}$ & 2.54601500 & 0.01559100 & -1.56100200 \\
\hline C -0.58113800 & 2.54751400 & -2.88685400 & & & & \\
\hline C -1.85699800 & 2.55567800 & -3.43668000 & 10 & & & \\
\hline C - 2.82118000 & 1.70646500 & -2.90959700 & & -1.97453700 & -1.41577300 & -0.78751100 \\
\hline C - 2.49448200 & 0.86224900 & -1.84962700 & $\mathrm{H}$ & -2.45409400 & -2.81432900 & -0.61875900 \\
\hline C -0.68999600 & 0.83949900 & 1.31960400 & $\mathrm{H}$ & -1.55199100 & -1.17547700 & -2.18923000 \\
\hline C -1.81895300 & 1.55702500 & 1.72759400 & $\mathrm{C}$ & -3.32788900 & -0.25214500 & -0.25226700 \\
\hline C -1.82526300 & 2.48764600 & 2.75881600 & $\mathrm{C}$ & -4.37715900 & -0.71103200 & 0.56706200 \\
\hline C -0.64360600 & 2.74911000 & 3.44557500 & $\mathrm{C}$ & -3.33522200 & 1.10019100 & -0.64791000 \\
\hline C $\quad 0.50823200$ & 2.06528500 & 3.08481400 & $\mathrm{C}$ & -5.39221100 & 0.15098700 & 0.98482500 \\
\hline C $\quad 0.46242300$ & 1.13687200 & 2.04491800 & $\mathrm{H}$ & -4.40842300 & -1.75341300 & 0.87767000 \\
\hline F - 1.34398500 & -2.09356200 & -2.04570100 & $\mathrm{C}$ & -4.34844300 & 1.96322700 & -0.23237800 \\
\hline F - 2.83685300 & -4.30762600 & -1.88246000 & $\mathrm{H}$ & -2.54771400 & 1.48397400 & -1.29360600 \\
\hline F -4.10431500 & -4.95141600 & 0.45874500 & $\mathrm{C}$ & -5.37707700 & 1.48886000 & 0.58623000 \\
\hline F -3.82431800 & -3.31848100 & 2.63488500 & $\mathrm{H}$ & -6.19546600 & -0.22113100 & 1.61505900 \\
\hline F - 2.33891800 & -1.12804400 & 2.51153200 & $\mathrm{H}$ & -4.34157500 & 3.00237900 & -0.55031300 \\
\hline F $\quad 0.96068600$ & 1.77686600 & -1.32960600 & $\mathrm{H}$ & -6.16873700 & 2.16050800 & 0.90753000 \\
\hline F $\quad 0.36591700$ & 3.36984400 & -3.37182500 & $\mathrm{~N}$ & 2.81526000 & -1.42926000 & 0.03304300 \\
\hline F - 2.15248700 & 3.37355600 & -4.45640200 & $\mathrm{C}$ & 3.27227500 & -0.05122000 & 0.03132300 \\
\hline F - -4.06168800 & 1.70757300 & -3.42313800 & $\mathrm{C}$ & 2.31870700 & 0.96808800 & -0.03523200 \\
\hline F -3.50150700 & 0.08672700 & -1.39805500 & $\mathrm{C}$ & 4.63938800 & 0.22671200 & 0.10297900 \\
\hline F $\quad 1.64471000$ & 0.51868500 & 1.78197600 & $\mathrm{C}$ & 2.75051600 & 2.29361300 & -0.04290600 \\
\hline F $\quad 1.66097900$ & 2.30025300 & 3.73639500 & $\mathrm{H}$ & 1.26035200 & 0.72559200 & -0.04959800 \\
\hline F - 0.62066200 & 3.64473900 & 4.44229100 & $\mathrm{C}$ & 5.05256800 & 1.55796700 & 0.09050700 \\
\hline F - 2.95175500 & 3.13432400 & 3.09762000 & $\mathrm{H}$ & 5.37405500 & -0.57098000 & 0.13861000 \\
\hline F - 2.99928000 & 1.34223300 & 1.10580600 & $\mathrm{C}$ & 4.11345300 & 2.58957700 & 0.01846200 \\
\hline $\mathrm{N} \quad 4.41337000$ & 0.04568900 & -0.70376100 & $\mathrm{H}$ & 2.01715300 & 3.09320200 & -0.08438300 \\
\hline C $\quad 5.68106800$ & -0.62306500 & -0.48829000 & $\mathrm{H}$ & 6.11299900 & 1.78642900 & 0.13287100 \\
\hline C $\quad 6.86895900$ & 0.06609300 & -0.74108300 & $\mathrm{H}$ & 4.44448300 & 3.62362700 & 0.01536300 \\
\hline C $\quad 5.68604700$ & -1.94192800 & -0.02973600 & $\mathrm{C}$ & 3.36776100 & -2.33569600 & 1.06320100 \\
\hline C 8.08405400 & -0.58714400 & -0.54013100 & $\mathrm{H}$ & 3.20852600 & -1.88526900 & 2.04487100 \\
\hline H $\quad 6.84592500$ & 1.08711000 & -1.10885800 & $\mathrm{H}$ & 2.86618600 & -3.30031800 & 0.99451000 \\
\hline C 6.91069600 & -2.58169700 & 0.16710400 & $\mathrm{H}$ & 4.43747700 & -2.46637300 & 0.89033500 \\
\hline
\end{tabular}




\begin{tabular}{|c|c|c|c|c|c|c|}
\hline $\mathrm{C}$ & 1.91483200 & -1.83064900 & -0.79282100 & F -6.48628400 & 1.12151100 & -1.60782100 \\
\hline 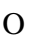 & -0.52774600 & -1.20005500 & 0.07276500 & F - 4.37729200 & 1.56343300 & -0.05969500 \\
\hline $\mathrm{H}$ & 1.55305700 & -2.85028200 & -0.73809000 & $\mathrm{~N} \quad 5.55364300$ & -1.93491500 & 0.04905400 \\
\hline 11 & -0.63710200 & -1.20148000 & 1.03819200 & C 6.66321300 & -1.04900900 & -0.16269500 \\
\hline \multirow[t]{2}{*}{$\mathrm{H}$} & 1.56048900 & -1.16145400 & -1.56575800 & C 7.00540700 & -0.12599300 & 0.83153700 \\
\hline & & & & C 7.39601000 & -1.11472000 & -1.35235600 \\
\hline \multicolumn{2}{|c|}{ TS14 } & & & C 8.07382100 & 0.74532900 & 0.62372200 \\
\hline \multicolumn{2}{|c|}{ C -0.42181200} & 0.25568700 & -2.25451400 & H $\quad 6.45442700$ & -0.10828200 & 1.76751000 \\
\hline \multicolumn{2}{|c|}{ O -0.50639000 } & -0.00490900 & -1.00366700 & C 8.47104400 & -0.24668300 & -1.54322600 \\
\hline \multicolumn{2}{|c|}{ O $\quad 0.65885200$} & 0.24428900 & -2.88131000 & H $\quad 7.12304300$ & -1.82429800 & -2.12658400 \\
\hline \multicolumn{2}{|c|}{ H - 1.33356500} & 0.50049200 & -2.80832900 & C 8.80984200 & 0.68620000 & -0.56132100 \\
\hline \multicolumn{2}{|c|}{ H $\quad 2.57944600$} & -1.77464100 & 0.93027300 & H $\quad 8.33909800$ & 1.45906700 & 1.39814900 \\
\hline \multicolumn{2}{|c|}{ B -1.77225400 } & -0.03817300 & -0.10417200 & H $\quad 9.03744200$ & -0.29580100 & -2.46871400 \\
\hline \multicolumn{2}{|c|}{ C - 1.72497600} & 1.36607300 & 0.74721800 & H $\quad 9.64666000$ & 1.36087200 & -0.71677800 \\
\hline \multicolumn{2}{|c|}{ C - 1.87025700} & 1.47805600 & 2.13068100 & C $\quad 5.63458400$ & -3.33433600 & -0.39108600 \\
\hline \multicolumn{2}{|c|}{ C - 1.79063400} & 2.68759300 & 2.81967600 & H $\quad 6.67697000$ & -3.64893600 & -0.35079900 \\
\hline \multicolumn{2}{|c|}{ C - 1.56384700} & 3.86408500 & 2.11853300 & Н $\quad 5.04247000$ & -3.96035900 & 0.27719600 \\
\hline \multicolumn{2}{|c|}{ C - 1.42746700} & 3.81199300 & 0.73580400 & Н $\quad 5.25244000$ & -3.43998100 & -1.41352500 \\
\hline \multicolumn{2}{|c|}{ C - 1.51808600} & 2.58339200 & 0.09227700 & C 4.36538200 & -1.40739700 & 0.34264000 \\
\hline \multicolumn{2}{|c|}{ C - 1.53504400} & -1.36541500 & 0.85459700 & O 3.41045500 & -2.24559000 & 0.71785800 \\
\hline \multicolumn{2}{|c|}{ C -0.28533100} & -1.73619000 & 1.35048100 & H 4.34097700 & -0.37401200 & 0.67477000 \\
\hline \multicolumn{2}{|c|}{ C -0.04219000} & -2.85700900 & 2.13517000 & Si 2.44778100 & -0.29768300 & -1.96887800 \\
\hline \multicolumn{2}{|c|}{ C - 1.09966900} & -3.68084900 & 2.49441200 & H $\quad 1.66963200$ & -1.41171700 & -1.38367200 \\
\hline \multicolumn{2}{|c|}{ C - 2.37641900} & -3.34645200 & 2.06170500 & H $\quad 2.98000000$ & -0.46343500 & -3.34128000 \\
\hline \multicolumn{2}{|c|}{ C - 2.56642100} & -2.21267100 & 1.27402800 & H 3.77417600 & -0.84403700 & -1.20039700 \\
\hline \multicolumn{2}{|c|}{ C -3.11055300 } & -0.20972000 & -1.04545500 & C 2.62024600 & 1.44448600 & -1.26312400 \\
\hline \multicolumn{2}{|c|}{ C -4.27238400} & 0.55917800 & -0.95471900 & C 2.39108900 & 1.76630200 & 0.08741500 \\
\hline \multicolumn{2}{|c|}{ C -5.39963700 } & 0.34596000 & -1.74690200 & C 3.06722000 & 2.47250200 & -2.11409700 \\
\hline \multicolumn{2}{|c|}{ C -5.40059400 } & -0.67987300 & -2.68293600 & C $\quad 2.59668800$ & 3.06272200 & 0.56670500 \\
\hline & -4.27041500 & -1.47944100 & -2.81241300 & H $\quad 2.02187000$ & 1.01058200 & 0.77503100 \\
\hline & -3.17236600 & -1.23271400 & -1.99657000 & C $\quad 3.27693400$ & 3.76791800 & -1.63767400 \\
\hline & 2.11025500 & 0.39168200 & 2.89859800 & H $\quad 3.25068000$ & 2.26499700 & -3.16611700 \\
\hline & 1.93225300 & 2.72261500 & 4.15527300 & C 3.04188200 & 4.06706500 & -0.29449500 \\
\hline & 1.48286200 & 5.03577200 & 2.76408100 & H $\quad 2.40172000$ & 3.28886700 & 1.61228900 \\
\hline & -1.22360800 & 4.94172600 & 0.04051300 & H 3.61913800 & 4.54449400 & -2.31728700 \\
\hline & -1.41597900 & 2.61443700 & -1.25855000 & H 3.19954600 & 5.07624600 & 0.07725100 \\
\hline & 0.82809000 & -0.97302700 & 1.10248400 & & & \\
\hline & 1.20986600 & -3.13736100 & 2.54539300 & TS15 & & \\
\hline & -0.89443800 & -4.76648500 & 3.24989900 & C -0.70622700 & 0.81226400 & -2.25429900 \\
\hline & -3.41920600 & -4.11431200 & 2.41042900 & O - 0.71901900 & 0.45784600 & -1.01071700 \\
\hline & -3.84813100 & -1.95899600 & 0.93728000 & O $\quad 0.33451100$ & 1.03287300 & -2.88234800 \\
\hline & 2.10371600 & -2.05008900 & -2.16620800 & H - 1.67688500 & 0.92536800 & -2.75840800 \\
\hline & 4.25229200 & -2.47569200 & -3.71186100 & B -1.89305000 & 0.05121200 & -0.11197500 \\
\hline & 6.47437300 & -0.89486700 & -3.45338100 & C -2.19581800 & 1.30718700 & 0.90975400 \\
\hline
\end{tabular}




\begin{tabular}{|c|c|c|c|c|c|c|}
\hline C - 2.69951800 & 1.11342500 & 2.19871600 & $\mathrm{C}$ & 4.69493900 & -2.84377300 & 0.36811000 \\
\hline C -2.88461000 & 2.13601100 & 3.12387200 & $\mathrm{H}$ & 5.39464300 & -3.48307300 & 0.90760600 \\
\hline C -2.56944300 & 3.44319300 & 2.77011200 & $\mathrm{H}$ & 3.77376800 & -2.73267900 & 0.93953800 \\
\hline C - 2.09556900 & 3.69667000 & 1.49021000 & $\mathrm{H}$ & 4.46851400 & -3.28279700 & -0.60795000 \\
\hline C - 1.94839900 & 2.64263600 & 0.59173700 & $\mathrm{C}$ & 4.57008600 & -0.44094800 & 0.21937800 \\
\hline C -1.23766200 & -1.21238900 & 0.74199100 & $\mathrm{H}$ & 5.03462800 & 0.52854800 & 0.09380900 \\
\hline C $\quad 0.06047400$ & -1.11864700 & 1.25274100 & & 2.48197700 & 0.42090900 & -2.08102900 \\
\hline C $\quad 0.71617500$ & -2.16212500 & 1.89705300 & $\mathrm{H}$ & 1.64062600 & -0.56599800 & -1.38294200 \\
\hline C $\quad 0.06525700$ & -3.37153000 & 2.09608000 & $\mathrm{H}$ & 2.68520300 & 0.20044800 & -3.53226800 \\
\hline C -1.24322800 & -3.50273400 & 1.65013400 & $\mathrm{H}$ & 3.86049500 & -0.12562000 & -1.55471400 \\
\hline C -1.86001000 & -2.43597600 & 0.99772800 & $\mathrm{C}$ & 2.68563400 & 2.18796900 & -1.45424300 \\
\hline C -3.18163800 & -0.39590900 & -1.03262300 & $\mathrm{C}$ & 1.70168500 & 2.86903800 & -0.71959700 \\
\hline C -4.48828500 & 0.07634500 & -0.89703900 & $\mathrm{C}$ & 3.88881000 & 2.86559400 & -1.72934600 \\
\hline C -5.54967800 & -0.35228600 & -1.69480300 & $\mathrm{C}$ & 1.90210100 & 4.18073900 & -0.28497300 \\
\hline C -5.32875100 & -1.29832100 & -2.68598700 & $\mathrm{H}$ & 0.76789300 & 2.37374200 & -0.48986100 \\
\hline C -4.04491100 & -1.80258700 & -2.86531300 & $\mathrm{C}$ & 4.09229300 & 4.17945700 & -1.30180800 \\
\hline C -3.02089000 & -1.34976500 & -2.04316000 & $\mathrm{H}$ & 4.67681400 & 2.36992800 & -2.29475400 \\
\hline F - 3.05245100 & -0.12514300 & 2.61093400 & $\mathrm{C}$ & 3.09702700 & 4.84047800 & -0.57782900 \\
\hline F - 3.36425000 & 1.87389500 & 4.35123300 & $\mathrm{H}$ & 1.11400600 & 4.68713900 & 0.26736000 \\
\hline F - 2.72660900 & 4.44306700 & 3.64937600 & $\mathrm{H}$ & 5.02409200 & 4.68811900 & -1.53720500 \\
\hline F - 1.77775100 & 4.95234400 & 1.12581700 & $\mathrm{H}$ & 3.25170500 & 5.86462500 & -0.24754900 \\
\hline F - 1.52719800 & 2.99717500 & -0.64752300 & $\mathrm{H}$ & 3.56217100 & -0.50895300 & 0.61297100 \\
\hline F $\quad 0.77453100$ & 0.02803500 & 1.14705600 & & & & \\
\hline F $\quad 1.99537500$ & -2.00862500 & 2.31502300 & \multicolumn{2}{|c|}{ TS16 } & & \\
\hline F $\quad 0.68591700$ & -4.38831700 & 2.71224600 & B & 2.22759100 & -0.16874700 & -0.08886600 \\
\hline F - 1.90232100 & -4.65585500 & 1.84865100 & $\mathrm{C}$ & 2.24798200 & 1.41200500 & -0.24620800 \\
\hline F - 3.13543500 & -2.65709900 & 0.61592300 & $\mathrm{C}$ & 1.55520700 & 2.05214200 & -1.28173100 \\
\hline F - 1.79581100 & -1.88559500 & -2.26428700 & $\mathrm{C}$ & 1.50889900 & 3.42988500 & -1.44336200 \\
\hline F -3.81090900 & -2.71770000 & -3.82023300 & $\mathrm{C}$ & 2.21093900 & 4.23936400 & -0.55539400 \\
\hline F - 6.33635300 & -1.71863000 & -3.46256400 & $\mathrm{C}$ & 2.93260200 & 3.65567500 & 0.47956300 \\
\hline F -6.78388200 & 0.14385400 & -1.50969500 & $\mathrm{C}$ & 2.93596400 & 2.27085800 & 0.61916400 \\
\hline F - 4.80965000 & 0.99411600 & 0.03932600 & $\mathrm{C}$ & 2.44487900 & -1.08036500 & -1.37545400 \\
\hline $\mathrm{N} \quad 5.30736900$ & -1.51381700 & 0.18402200 & $\mathrm{C}$ & 1.89243600 & -2.36266700 & -1.47327100 \\
\hline C $\quad 6.70741000$ & -1.44641800 & -0.16084200 & $\mathrm{C}$ & 2.05836900 & -3.19112000 & -2.57531800 \\
\hline C 7.51367100 & -0.47651000 & 0.44272000 & $\mathrm{C}$ & 2.83645500 & -2.75205000 & -3.64254000 \\
\hline C $\quad 7.24030300$ & -2.35527400 & -1.07917300 & $\mathrm{C}$ & 3.42161800 & -1.49216200 & -3.59008200 \\
\hline C 8.86381200 & -0.40570500 & 0.10366500 & $\mathrm{C}$ & 3.21703600 & -0.68630500 & -2.47363800 \\
\hline H 7.09848300 & 0.19600100 & 1.18766300 & $\mathrm{C}$ & 2.48617800 & -0.81444300 & 1.34302900 \\
\hline C 8.59326500 & -2.27384100 & -1.40704100 & $\mathrm{C}$ & 3.41453800 & -1.83952400 & 1.55869100 \\
\hline Н 6.61031900 & -3.10209000 & -1.55039600 & $\mathrm{C}$ & 3.65321900 & -2.40184000 & 2.80972000 \\
\hline C $\quad 9.40534000$ & -1.30132000 & -0.82064000 & $\mathrm{C}$ & 2.94184700 & -1.94546600 & 3.91304500 \\
\hline H $\quad 9.49352700$ & 0.34272600 & 0.57518900 & $\mathrm{C}$ & 2.00645500 & -0.92742500 & 3.75184200 \\
\hline H $\quad 9.00892700$ & -2.97170300 & -2.12762700 & $\mathrm{C}$ & 1.81142800 & -0.38119200 & 2.49028800 \\
\hline Н 10.45890100 & -1.24601800 & -1.07803500 & $\mathrm{~F}$ & 0.85503400 & 1.31780000 & -2.17177800 \\
\hline
\end{tabular}




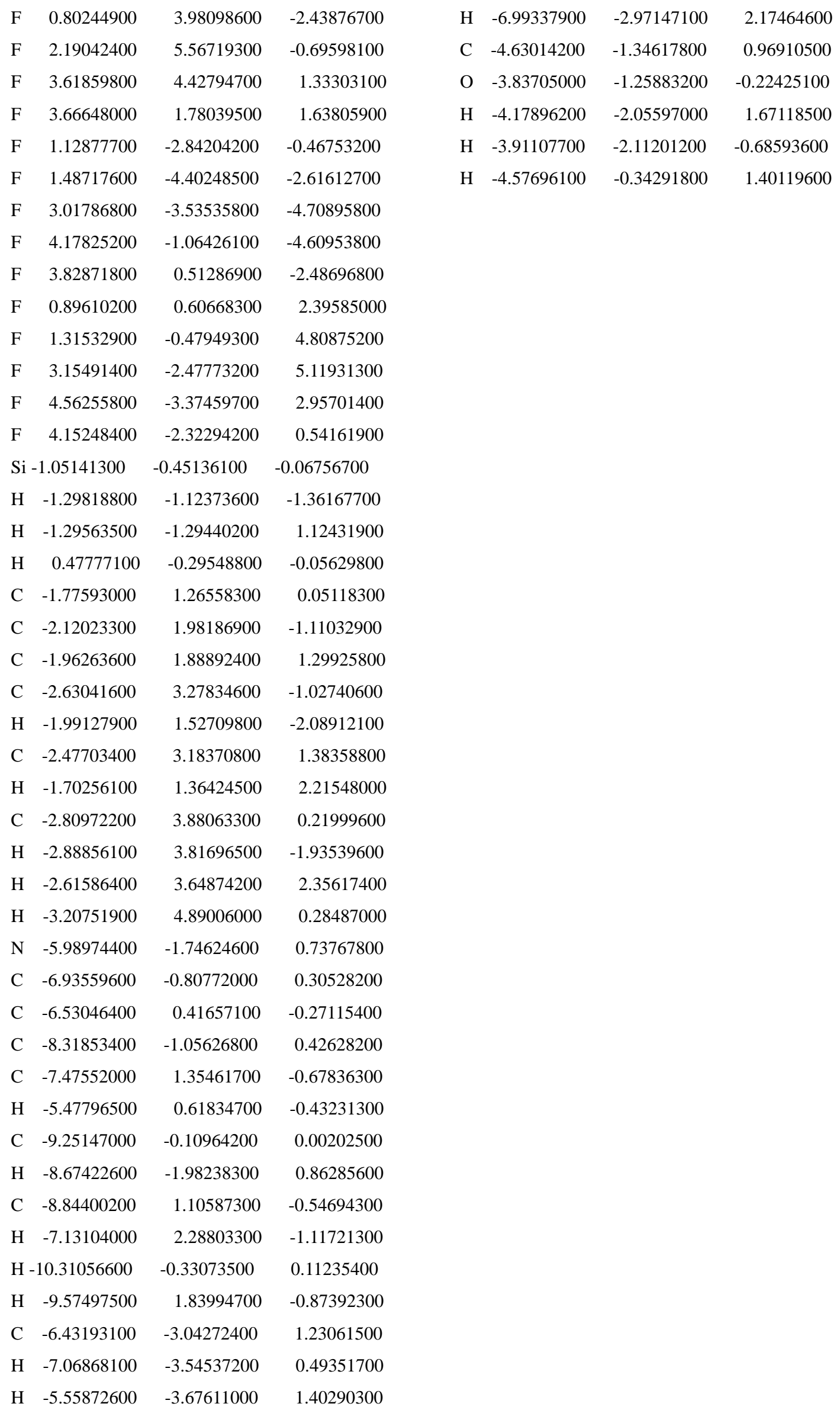

